\title{
The odd-dimensional analogue of a theorem of Getzler and Wu
}

\author{
Zhizhang Xie*
}

\begin{abstract}
We prove an analogue for odd-dimensional manifolds with boundary, in the bcalculus setting, of the higher Atiyah-Patodi-Singer index theorem by Getzler and by $\mathrm{Wu}$, and thus obtain a natural counterpart of the eta invariant for even-dimensional closed manifolds.
\end{abstract}

Mathematics Subject Classification (2010). 58Jxx, 46L80; 58B34, 46L87.

Keywords. Odd-dimensional manifolds with boundary, b-calculus, noncommutative geometry, Connes-Chern character, odd APS index.

\section{Introduction}

The goal of this paper is to prove an analogue for odd-dimensional manifolds with boundary of the higher Atiyah-Patodi-Singer index theorem of Getzler [Get93a] and $\mathrm{Wu}$ [Wu93]. For notational simplicity, we will restrict the discussion mainly to spin manifolds. However all results can be straightforwardly extended to general manifolds, with appropriate modification.

Suppose $N$ is an odd-dimensional spin manifold with boundary and carries an exact b-metric [Me193], cf. Section 1. For $g \in U_{k}\left(C^{\infty}(N)\right)$ a unitary over $N$, let Ch. $(g)$ (resp. $\mathrm{Ch}_{\bullet}^{\mathrm{dR}}(g)$ ) be the Chern character of $g$ in entire cyclic homology of $C^{\infty}(N)$ (resp. de Rham cohomology of $N$ ). In the following, $f_{N}$ is the regularized integral on $N$ with respect to its b-metric (see Section 1) and $\hat{A}(N)$ is the $\hat{A}$-genus form of $N$. Let $D$ be the Dirac operator on $N$ and ${ }^{\partial} D$ be its restriction to the boundary $\partial N$. Denote the higher eta cochain of ${ }^{\partial} D$ by $\eta^{\bullet}\left({ }^{\partial} D\right)$, introduced by Wu [Wu93].

Theorem. Let $N$ be an odd-dimensional spin manifold with boundary. Endow $N$ with an exact b-metric and let $D$ be its associated Dirac operator. Assume ${ }^{\partial} D$ is invertible. For $g \in U_{k}\left(C^{\infty}(N)\right)$ a unitary over $N$, if $\left\|d^{\partial} g\right\|<\lambda$ where $\lambda$ the lowest nonzero eigenvalue of $\left.\right|^{\partial} D \mid$ and ${ }^{\partial} g$ is the restriction of $g$ to the boundary, then

$$
\operatorname{sf}\left(D, g^{-1} D g\right)=f_{N} \hat{A}(N) \wedge \mathrm{Ch}_{\bullet}^{\mathrm{dR}}(g)-\left\langle\eta^{\bullet}\left({ }^{\partial} D\right), \mathrm{Ch} \bullet\left({ }^{\partial} g\right)\right\rangle .
$$

\footnotetext{
${ }^{*}$ The author was partially supported by the US National Science Foundation awards no. DMS-0969672.
} 
Here $\operatorname{sf}\left(D, g^{-1} D g\right)$ is the spectral flow of the path $D_{u}=(1-u) D+u g^{-1} D g$ with $u \in[0,1]$ (see Section 4). In order for $\operatorname{sf}\left(D, g^{-1} D g\right)$ to be well defined, the infimum of the essential spectrum of $\left|D_{u}\right|$, denoted by inf $\operatorname{spec}_{\text {ess }}\left(\left|D_{u}\right|\right)$, has to be greater than zero for each $u$. The latter condition is fulfilled if and only if the restriction $D_{u}$ to the boundary $\partial N$ is invertible for each $u$. Thus the almost flatness condition $\left\|d^{\partial} g\right\|<\lambda$ ensures that $\operatorname{sf}\left(D, g^{-1} D g\right)$ is well defined.

Let ${ }^{\mathrm{b}} \mathrm{Ch}^{\bullet}\left(D_{t}\right)$ be the b-analogue of the odd Chern character by Jaffe-LesniewskiOsterwalder [JLO88], cf. Section 2. The above theorem is proved by interpolating between the limit of ${ }^{\mathrm{b}} \mathrm{Ch}^{\circ}\left(D_{t}\right)$ as $t \rightarrow \infty$ and its limit as $t \rightarrow 0$, where $D_{t}=t D$. In fact, the limit at $t=\infty$ does not exist in general. However, when evaluated at Ch. $(g)$ with $g$ satisfying the almost flat condition above, the limit of ${ }^{\mathrm{b}} \mathrm{Ch}^{\bullet}\left(D_{t}\right)$ as $t \rightarrow \infty$ does exist and gives the spectral flow $\operatorname{sf}\left(D, g^{-1} D g\right)$. To prove this, i.e., the equality

$$
\lim _{t \rightarrow \infty}\left\langle{ }^{\mathrm{b}} \mathrm{Ch}^{\bullet}\left(D_{t}\right), \mathrm{Ch}_{\bullet}(g)\right\rangle=\operatorname{sf}\left(D, g^{-1} D g\right),
$$

we first show (see Proposition 4.6 below) that

$$
\operatorname{sf}\left(D, g^{-1} D g\right)=\lim _{\varepsilon \rightarrow \infty} \frac{\varepsilon}{\sqrt{\pi}} \int_{0}^{1}{ }^{\mathrm{b}} \operatorname{Tr}\left(\dot{D}_{u} e^{-\varepsilon^{2} D_{u}^{2}}\right) d u .
$$

This is a generalization to the b-calculus setting of Getzler's spectral flow formula for closed manifolds, cf. [Get93b], Corollary 2.7. Once we show eq. (0.3), the proof of eq. (0.2) reduces to

$$
\lim _{t \rightarrow \infty} \frac{t}{\sqrt{\pi}} \int_{0}^{1}{ }^{\mathrm{b}} \operatorname{Tr}\left(\dot{D}_{u} e^{-t^{2} D_{u}^{2}}\right) d u=\lim _{t \rightarrow \infty}\left\langle{ }^{\mathrm{b}} \mathrm{Ch}^{\bullet}\left(D_{t}\right), \mathrm{Ch} \cdot(g)\right\rangle .
$$

In turn, to verify this, we consider a multiparameter version of the Chern character $\mathrm{Ch}(\mathbb{A})$ of the superconnection $\mathbb{A}$ (see [Get93b], also Section 5 below, for the precise definition). Each side of eq. (0.4) corresponds to one term in the formula obtained by applying Stokes theorem to $\mathrm{Ch}\left(\mathbb{A}_{t}\right)$ for each fixed $t$. We then show the vanishing of the rest of the terms as $t \rightarrow \infty$, hence prove the validity of eq. (0.4), cf. Section 5 . The rest of the proof proceeds along the lines of Getzler's even counterpart, cf. [Get93a].

Due to the fact that ${ }^{\mathrm{b}} \mathrm{Tr}$ is not a trace, ${ }^{\mathrm{b}} \mathrm{Ch}^{\circ}\left(D_{t}\right)$ is not a closed cochain. The integral of its boundary from 0 to $\infty$ gives the odd eta cochain $\eta^{\bullet}\left({ }^{\partial} D\right)$ on the right-hand side of $(0.1)$.

As a corollary of the main theorem, by comparing eq. (0.1) with Dai-Zhang's Toeplitz index formula for odd-dimensional manifolds with boundary [DZ06], we show that if $\left\|d^{\partial} g\right\|<\lambda$, then

$$
\left\langle\eta^{\bullet}\left({ }^{\partial} D\right), \mathrm{Ch}_{\bullet}\left({ }^{\partial} g\right)\right\rangle=-\bar{\eta}\left(\partial N,{ }^{\partial} g\right) \bmod \mathbb{Z},
$$

where $\bar{\eta}\left(\partial N,{ }^{\partial} g\right)$ is the (reduced) eta invariant of Dai-Zhang. This equality provides more evidence for the naturality of the Dai-Zhang eta invariant for even-dimensional closed manifolds. 
An outline of this article is as follows. In Section 1, we recall some facts from b-calculus on manifolds with boundary and Chern characters in cyclic homology. In Section 2, we define a b-analogue of the JLO Chern character and prove its entireness. Then we state our main theorem (Theorem 3.1 below) in Section 3. We prove the main step of the proof to the main theorem in Section 4 and 5.

Acknowledgements. I am grateful to Henri Moscovici for his continuous support and advice. I also want to thank Matthias Lesch for many helpful suggestions.

\section{Preliminaries}

Throughout the paper, we denote by $\mathrm{Cl}_{q}(\mathbb{C})$ the complex Clifford algebra with odddegree generators $c_{i}, 1 \leq i \leq q$, and relations

$$
c_{i} c_{j}+c_{j} c_{i}=-2 \delta_{i j} .
$$

This is a $\mathbb{Z}_{2}$-graded $*$-algebra with $c_{i}^{*}=-c_{i}$.

1.1. Manifolds with Boundary and b-metrics. Let $M$ be an odd-dimensional spin manifold with boundary. We fix a Riemannian metric, say $w$, and a spin structure on $M$. Furthermore, we assume the Riemannian metric is of product type near the boundary, that is, on $[0, \varepsilon)_{x} \times \partial M$ a collar neighborhood of $\partial M$, it takes the form

$$
w=(d x)^{2}+h,
$$

where $h$ is the Riemannian metric on $\partial M$. Denote by $\hat{M}$ the manifold obtained by attaching an infinite cylinder $(-\infty, 0] \times \partial M$ to $M$ along $\partial M$ :

$$
\widehat{M}=(-\infty, 0] \times \partial M \cup_{\partial M} M .
$$

The Riemannian metric $M$ extends naturally to a Riemannian metric on $\widehat{M}$, still denoted by $w$.

Notice that $(\hat{M}, w)$ is isometric to a standard b-manifold, that is, a manifold with boundary carrying a b-metric. To see this, one performs the change of variable $x \mapsto r=e^{x}$ on the cylindrical end. This replaces $(-\infty, 0]_{x} \times \partial M$ by a compact cylinder $[0,1]_{r} \times \partial M$. Moreover, the metric $w$ induces a metric on $N=[0,1] \times$ $\partial M \cup_{\partial M} M$ under the coordinate change. In particular, the induced metric restricted on $[0,1]_{r} \times \partial M$ takes the form

$$
\left(\frac{d r}{r}\right)^{2}+h
$$

which is an exact b-metric on $N$, cf. [Me193] and [Loy05]. Unless otherwise specified, all $b$-metrics in this paper are assumed to be exact. 
1.2. Clifford modules and Dirac operators. Consider $N=[0,1]_{r} \times \partial M \cup_{\partial M} M$ with an exact b-metric. The set of b-vector fields, that is, vector fields on $N$ tangential to $\partial N$, is closed under Lie bracket. By the Swan-Serre Theorem, such vector fields are smooth sections of a vector bundle ${ }^{b} T N$ over $N$, called the b-tangent bundle of $N$, cf. [Me193], Lemma 2.5. We denote its dual bundle, the b-cotangent bundle, by ${ }^{b} T^{*} N$.

By a Clifford module over $N$ of degree $q$, we mean a $\mathbb{Z}_{2}$-graded Hermitian vector bundle $\mathcal{E}$ over $N$ with commuting graded $*$-actions of the Clifford algebra $\mathrm{Cl}_{q}(\mathbb{C})$ and the Clifford bundle $\mathrm{Cl}\left({ }^{b} T^{*} N\right)$, cf. [Get93a].

Let $S$ be the spinor bundle over $N$ and $\mathbb{C}^{1 \mid 1}=\mathbb{C}^{+} \oplus \mathbb{C}^{-}$be a $\mathbb{Z}_{2}$-graded two dimensional vector bundle. Then $S \otimes \mathbb{C}^{1 \mid 1}$ is a Clifford module of degree 1 over $N$, where each $\left.\omega \in \Gamma\left(N, \mathrm{Cl}^{b} T^{*} N\right)\right)$ acts on $S \otimes \mathbb{C}^{1 \mid 1}$ by $\left(\begin{array}{cc}0 & c(\omega) \\ c(\omega) & 0\end{array}\right)$ and the generator $e_{1}$ of $\mathrm{Cl}_{1}(\mathbb{C})$ acts on $S \otimes \mathbb{C}^{1 \mid 1}$ by $\left(\begin{array}{cc}0 & 1 \\ -1 & 0\end{array}\right)$.

1.3. b-norm. In this section, we introduce a b-norm on $C_{\exp }^{\infty}(\hat{M})$. We shall use this b-norm to prove the entireness of the b-JLO Chern character in Section 2. Here $C_{\exp }^{\infty}(\hat{M})$ is the space of smooth functions on $\hat{M}$ which expands exponentially on the infinite cylinder $(-\infty, 0]_{x} \times \partial M$, cf. [Loy05]. A smooth function $f \in C^{\infty}(\hat{M})$ expands exponentially on $(-\infty, 0]_{x} \times \partial M$ if

$$
f(x, y) \sim \sum_{k=0}^{\infty} e^{k x} f_{k}(y)
$$

for $(x, y) \in(-\infty, 0]_{x} \times \partial M$, where $f_{k}(y) \in C^{\infty}(\partial M)$ for each $k$. More precisely, we have

$$
f(x, y)-\sum_{k=0}^{N-1} e^{k x} f_{k}(y)=e^{N x} R_{N}(x, y),
$$

where all derivatives of $R_{N}(x, y)$ in $x$ and $y$ are bounded.

Remark 1.1. Notice that $C_{\exp }^{\infty}(\hat{M})$ becomes exactly $C^{\infty}(N)$ if one performs the change of variable $x \rightarrow e^{x}$ on the cylindrical end.

On $(-\infty, 0]_{x} \times \partial M$, for each $a \in C_{\exp }^{\infty}(\hat{M})$, we have

$$
a=a_{c}+e^{x} a_{\infty},
$$

where $a_{c}, a_{\infty} \in C_{\exp }^{\infty}(\hat{M})$ and $a_{c}$ is constant with respect to $x$. We define a norm on $C_{\exp }^{\infty}(\hat{M})$ by

$$
{ }^{\mathrm{b}}\|a\|:=\|a\|_{1}+2\left\|a_{\infty}\right\|_{1}
$$

where $\|a\|_{1}$ is the $C^{1}$-norm of $a$ and $\left\|a_{\infty}\right\|_{1}$ is the $C^{1}$-norm of $a_{\infty}$.

Lemma 1.2. ${ }^{\mathrm{b}}\|\cdot\|$ is a well-defined multiplicative norm. 
Proof. Note that $(a+b)_{\infty}=a_{\infty}+b_{\infty}$ and $(a b)_{\infty}=a_{c} b_{\infty}+a_{\infty} b_{c}+e^{x} a_{\infty} b_{\infty}$. So it is clear that

$$
{ }^{\mathrm{b}}\|\lambda a\|=|\lambda| \cdot{ }^{\mathrm{b}}\|a\|, \quad{ }^{\mathrm{b}}\|a+b\| \leq{ }^{\mathrm{b}}\|a\|+{ }^{\mathrm{b}}\|b\| .
$$

To prove the norm is multiplicative, we first notice that $\left\|a_{c}\right\| \leq\|a\|,\left\|d a_{c}\right\| \leq\|d a\|$ and

$$
d\left(e^{x} a_{\infty} b_{\infty}\right)=\left(e^{x} d x\right) a_{\infty} b_{\infty}+e^{x} d\left(a_{\infty} b_{\infty}\right) .
$$

Thus we have

$$
\begin{aligned}
2 \| a_{c} b_{\infty} & +a_{\infty} b_{c}+e^{x} a_{\infty} b_{\infty} \|_{1} \\
= & 2\left\|a_{c} b_{\infty}+a_{\infty} b_{c}+e^{x} a_{\infty} b_{\infty}\right\|+2\left\|d\left(a_{c} b_{\infty}+a_{\infty} b_{c}+e^{x} a_{\infty} b_{\infty}\right)\right\| \\
& +2\|a\| \cdot\left\|d b_{\infty}\right\|+2\left\|d a_{\infty}\right\| \cdot\|b\| \\
\leq & 2\|a\|_{1} \cdot\left\|b_{\infty}\right\|_{1}+2\left\|a_{\infty}\right\|_{1} \cdot\|b\|_{1}+4\left\|a_{\infty}\right\|_{1} \cdot\left\|b_{\infty}\right\|_{1} .
\end{aligned}
$$

By applying the inequality $\|a b\|_{1} \leq(\|a\|+\|d a\|)(\|b\|+\|d b\|)$, we obtain

$$
{ }^{\mathrm{b}}\|a b\| \leq{ }^{\mathrm{b}}\|a\| \cdot{ }^{\mathrm{b}}\|b\| .
$$

1.4. b-trace. For $f \in C_{\exp }^{\infty}(\hat{M})$, we have

$$
f=f_{c}+e^{x} f_{\infty}
$$

on the cylindrical end $(-\infty, 0] \times \partial M$, where $f_{c}$ is constant with respect to $x$.

Definition 1.3. The regularized integral of $f \in C_{\exp }^{\infty}(\hat{M})$ with respect to the b-metric is defined to be

$$
f_{\widehat{M}} f d \mathrm{vol}:=\left.\int_{M} f\right|_{M} d \mathrm{vol}+\int_{(-\infty, 0] \times \partial M} e^{x} f_{\infty} d \mathrm{vol} .
$$

For $A \in{ }^{\mathrm{b}} \Psi^{-\infty}(\hat{M}, \mathcal{V})$, let $K_{A}$ be its Schwartz kernel and $\left.K_{A}\right|_{\Delta}$ the restriction of $K_{A}$ to the diagonal $\Delta \subset \hat{M} \times \hat{M}$. Then the fiberwise trace of $\left.K_{A}\right|_{\Delta}$, denoted by $\operatorname{tr}\left(\left.K_{A}\right|_{\Delta}\right)$, is a function in $C_{\exp }^{\infty}(\hat{M})$, cf. [Loy05]. We define the b-trace of $A \in$ ${ }^{\mathrm{b}} \Psi^{-\infty}(\hat{M}, \mathcal{V})$ to be

$$
{ }^{\mathrm{b}} \operatorname{Tr}(A):=f_{\widehat{M}} \operatorname{tr}\left(\left.K_{A}\right|_{\Delta}\right) d \text { vol. }
$$

When $\mathcal{V}$ is $\mathbb{Z}_{2}$-graded, we define the b-supertrace of $A$ by

$$
{ }^{\mathrm{b}} \operatorname{Str}(A)=f_{\widehat{M}} \operatorname{str}\left(\left.K_{A}\right|_{\Delta}\right) d \mathrm{vol},
$$

where str is the fiberwise supertrace on $\operatorname{End}_{\mathbb{Z}_{2}}(\mathcal{V})$. 
1.5. Cyclic homology and cyclic cohomology. For $A$ an algebra over $\mathbb{C}$, let

$$
C_{n}(A)=A \otimes(A / \mathbb{C})^{\otimes n} .
$$

An element of $C_{n}(A)$ is denoted by $\left(a_{0}, a_{1}, \ldots, a_{n}\right)$. Sometimes we also write $\left(a_{0}, a_{1}, \ldots, a_{n}\right)_{n}$ to emphasise the degree of the element.

\section{Definition 1.4.}

$$
\begin{aligned}
& b\left(a_{0}, \ldots, a_{n}\right)=\sum_{i=0}^{n}(-1)^{i}\left(a_{0}, \ldots, a_{i} a_{i+1}, \ldots, a_{n}\right), \\
& B\left(a_{0}, \ldots, a_{n}\right)=\sum_{i=0}^{n}(-1)^{n i}\left(1, a_{i}, \ldots, a_{n}, a_{0}, \ldots, a_{i-1}\right) .
\end{aligned}
$$

Let $C_{+}(A)=\prod_{k} C_{2 k}(A)$ and $C_{-}(A)=\prod_{k} C_{2 k+1}(A)$, then we have the chain map

$$
b+B: C_{ \pm}(A) \rightarrow C_{\mp}(A) .
$$

The homology of this chain complex is called the periodic cyclic homology of $A$, denoted by $\mathrm{HP}_{ \pm}(A)$.

When $A$ is a Banach algebra, we use the inductive tensor product instead of the algebraic tensor product in the definition of $C_{n}(A)$. We denote the resulted space of continuous even (resp. odd) chains by $C_{+}^{\text {top }}(A)$ (resp. $\left.C_{-}^{\text {top }}(A)\right)$. Let us define

$$
\left\|c_{0}+c_{1}+\cdots\right\|_{\lambda}=\sup _{n} \frac{\lambda^{n}}{\Gamma(n / 2)}\left\|c_{n}\right\| .
$$

Then an even chain $c_{0}+c_{2}+\cdots \in C_{+}^{\text {top }}(A)$ is called entire if $\left\|c_{0}+c_{2}+\cdots\right\|_{\lambda}$ is finite for some $\lambda>0$. Entire odd chains in $C_{-}^{\text {top }}(A)$ are defined the same way. The space of even (resp. odd) entire chains will be denoted by $C_{+}^{\omega}(A)$ (resp. $C_{-}^{\omega}(A)$ ). It is easy to check that $b$ and $B$ are continuous maps from $C_{ \pm}^{\omega}(A)$ to $C_{\mp}^{\omega}(A)$, hence $\left(C_{ \pm}^{\omega}(A), b+B\right)$ is a well-defined chain complex. The resulting homology is called the entire cyclic homology of $A$, denoted $H_{ \pm}^{\omega}(A)$.

Similarly, the entire cyclic cohomology of $A$, denoted by $H_{\omega}^{ \pm}$, is defined to be the homology of the cochain complex $\left(C_{\omega}^{ \pm}(A), b+B\right)$, where

$$
C_{\omega}^{ \pm}(A):=\left(C_{ \pm}^{\omega}(A)\right)^{*}=\text { the space of continuous linear functionals on } C_{ \pm}^{\omega}(A),
$$

with $b$ and $B$ being the obvious dual maps of those defined for cyclic homology.

1.6. Odd Chern character. For each Banach algebra $A$, we have the generalized trace map $\operatorname{Tr}: C_{n}\left(M_{r}(A)\right) \rightarrow C_{n}(A)$ with

$$
\operatorname{Tr}\left(a_{0}, \ldots, a_{n}\right)=\sum_{0 \leq i_{0}, \ldots, i_{n} \leq r}\left(\left(a_{0}\right)_{i_{0} i_{1}},\left(a_{1}\right)_{i_{1} i_{2}}, \ldots,\left(a_{n}\right)_{i_{n} i_{0}}\right) .
$$


It is easy to check that the generalized trace map induces a chain complex homomorphism $C_{ \pm}^{\omega}\left(M_{r}(A)\right) \rightarrow C_{ \pm}^{\omega}(A)$. For an invertible element $g \in \mathrm{GL}_{r}(A)$, its Chern character is defined to be

$$
\text { Ch. }(g):=\sum_{k=0}^{\infty}(-1)^{k} k ! \operatorname{Tr}\left(g^{-1}, g, \ldots, g^{-1}, g\right)_{2 k+1} .
$$

We have Ch. $(g) \in C_{-}^{\omega}(A)$ and $(b+B) C_{\bullet}(g)=0$. Moreover, let $h:[0,1] \rightarrow$ $\mathrm{GL}_{r}(A)$ be a smooth path of invertible elements. Then we have (cf. [Get93b])

$$
\frac{d}{d t} \mathrm{Ch}_{\bullet}\left(h_{t}\right)=(b+B) \widetilde{\mathrm{Ch}_{\bullet}}(h, t),
$$

where the secondary Chern character $\widetilde{\mathrm{Ch}_{\bullet}}(h, t)$ of $h$ is defined as

$$
\begin{gathered}
\widetilde{\mathrm{Ch}} .(h, t)=\operatorname{Tr}\left(h_{t}^{-1} \dot{h}_{t}\right)+\sum_{k=0}^{\infty}(-1)^{k+1} k ! \sum_{j=0}^{k} \operatorname{Tr}\left(h_{t}^{-1} \otimes h_{t}\right)^{\otimes(j+1)} \\
\left.\otimes h_{t}^{-1} \dot{h}_{t} \otimes\left(h_{t}^{-1} \otimes h_{t}\right)^{\otimes(k-j)}\right)_{2 k+2} .
\end{gathered}
$$

If we denote

$$
\operatorname{Tch}_{\bullet}(h)=\int_{0}^{1} \widetilde{\mathrm{Ch}_{\bullet}}(h, t) d t,
$$

then

$$
\text { Ch. }\left(h_{1}\right)-\text { Ch. }_{\bullet}\left(h_{0}\right)=(b+B) \text { Tch. }(h) \text {. }
$$

\section{JLO Chern character in b-calculus}

In this section, we shall define the b-JLO Chern character and prove its entireness. Let $\hat{M}$ be as before and $S$ be the spinor bundle over $\hat{M}$. Then $S_{1}=S \otimes \mathbb{C}^{1 \mid 1}$ is a Clifford module over $\hat{M}$ of degree 1 , where the generator $e_{1}$ of $\mathrm{Cl}_{1}(\mathbb{C})$ acts on $S_{1}$ by $\left(\begin{array}{cc}0 & 1 \\ -1 & 0\end{array}\right)$. Let $D$ be the Dirac operator on $\widehat{M}$ and write

$$
\mathfrak{D}=\left(\begin{array}{cc}
0 & D \\
D & 0
\end{array}\right) \in{ }^{\mathrm{b}} \Psi^{1}\left(\hat{M} ; S_{1}\right) .
$$

Notice that $\mathfrak{D}$ is odd and self-adjoint, and (graded) commutes with the action of $\mathrm{Cl}_{1}(\mathbb{C})$.

2.1. JLO Chern character in b-calculus. For $A \in{ }^{\mathrm{b}} \Psi^{m}\left(\widehat{M} ; \oint_{1}\right)$, we define

$$
{ }^{\mathrm{b}} \operatorname{Str}_{(1)}(A):=\frac{1}{2 \sqrt{\pi}}^{\mathrm{b}} \operatorname{Str}\left(e_{1} A\right) .
$$


More generally, for $A \in{ }^{\mathrm{b}} \Psi^{m}(\widehat{M} ; \mathcal{V})$ with $\mathcal{V}$ a Clifford module of degree $q$, we define

$$
{ }^{\mathrm{b}} \operatorname{Str}_{(q)}(A):=\frac{1}{(4 \pi)^{q / 2}}{ }^{\mathrm{b}} \operatorname{Str}\left(e_{1} \ldots e_{q} A\right),
$$

where $\left\{e_{1}, \ldots, e_{q}\right\}$ is a set of generators of $\mathrm{Cl}_{q}(\mathbb{C})$.

Definition 2.1. The b-JLO Chern character of $\mathfrak{D}$ is defined to be

$$
\begin{aligned}
{ }^{\mathrm{b}} \mathrm{Ch}^{n} & (\mathfrak{D})\left(a_{0}, \ldots, a_{n}\right) \\
& ={ }^{\mathrm{b}}\left\langle a_{0},\left[\mathfrak{D}, a_{1}\right], \ldots,\left[\mathfrak{D}, a_{n}\right]\right\rangle \\
& =\int_{\Delta^{n}}{ }^{\mathrm{b}} \operatorname{Str}_{(1)}\left(a_{0} e^{-\sigma_{0} \mathfrak{D}^{2}}\left[\mathfrak{D}, a_{1}\right] e^{-\sigma_{1} \mathfrak{D}^{2}} \ldots\left[\mathfrak{D}, a_{n}\right] e^{-\sigma_{n} \mathfrak{D}^{2}}\right) d \sigma,
\end{aligned}
$$

where $[-,-]$ stands for the graded commutator.

A straightforward calculation gives the following lemma.

Lemma 2.2. We have

$$
\begin{aligned}
{ }^{\mathrm{b}} \mathrm{Ch}^{2 k+1}(\mathfrak{D})\left(a_{0}, \ldots, a_{2 k+1}\right) \\
\quad=\frac{1}{\sqrt{\pi}} \int_{\Delta^{2 k+1}}{ }^{\mathrm{b}} \operatorname{Tr}\left(a_{0} e^{-\sigma_{0} D^{2}}\left[D, a_{1}\right] e^{-\sigma_{1} D^{2}} \ldots\left[D, a_{2 k+1}\right] e^{-\sigma_{2 k+1} D^{2}}\right) d \sigma .
\end{aligned}
$$

We see that the definition of ${ }^{b} \mathrm{Ch}^{\bullet}(\mathfrak{D})$ is a natural generalization of the JLO odd Chern character (for Dirac operators on closed manifolds) to the b-calculus setting.

Lemma 2.3 ([Get93b], Lemma 4.4). For $g \in U_{r}\left(C_{\exp }^{\infty}(\hat{M})\right)$, we have

$$
\left\langle{ }^{\mathrm{b}} \mathrm{Ch}^{\bullet}(\mathfrak{D}), \sum_{k=0}^{\infty} k ! \operatorname{Str}(p, \ldots, p)_{2 k+1}\right\rangle=\left\langle{ }^{\mathrm{b}} \mathrm{Ch}^{\bullet}(\mathfrak{D}), \mathrm{Ch}_{\bullet}\left(g^{-1}\right)-\mathrm{Ch}_{\bullet}(g)\right\rangle,
$$

where $p=\left(\begin{array}{cc}0 & g^{-1} \\ g & 0\end{array}\right) \in C_{\exp }^{\infty}(\hat{M}) \otimes \operatorname{End}\left(\mathbb{C}^{r \mid r}\right)$ with $\mathbb{C}^{r \mid r}=\left(\mathbb{C}^{r}\right)^{+} \oplus\left(\mathbb{C}^{r}\right)^{-}$being $\mathbb{Z}_{2}$-graded.

Proof. Notice that

$$
[\mathfrak{D}, p]=\left(\begin{array}{cc}
0 & {\left[\mathfrak{D}, g^{-1}\right]} \\
{[\mathfrak{D}, g]} & 0
\end{array}\right)
$$

and

$$
\begin{aligned}
& {[\mathfrak{D}, p] e^{-\sigma \mathfrak{D}^{2}}[\mathfrak{D}, p] e^{-\tau \mathfrak{D}^{2}}} \\
& \quad=\left(\begin{array}{cc}
-\left[\mathfrak{D}, g^{-1}\right] e^{-\sigma \mathfrak{D}^{2}}[\mathfrak{D}, g] e^{-\tau \mathfrak{D}^{2}} & 0 \\
0 & -[\mathfrak{D}, g] e^{-\sigma \mathfrak{D}^{2}}\left[\mathfrak{D}, g^{-1}\right] e^{-\tau \mathfrak{D}^{2}}
\end{array}\right) .
\end{aligned}
$$


It follows that

$$
\begin{aligned}
{ }^{\mathrm{b}}\langle p,[\mathfrak{D}, p], \ldots,[\mathfrak{D}, p]\rangle= & (-1)^{(k+1) \mathrm{b}}\left\langle g^{-1},[\mathfrak{D}, g], \ldots,\left[\mathfrak{D}, g^{-1}\right],[\mathfrak{D}, g]\right\rangle \\
& -(-1)^{(k+1) \mathrm{b}}\left\langle g,\left[\mathfrak{D}, g^{-1}\right], \ldots,[\mathfrak{D}, g],\left[\mathfrak{D}, g^{-1}\right]\right\rangle \\
= & (-1)^{k \mathrm{~b}}\left\langle g,\left[\mathfrak{D}, g^{-1}\right], \ldots,[\mathfrak{D}, g],\left[\mathfrak{D}, g^{-1}\right]\right\rangle \\
& -(-1)^{k \mathrm{~b}}\left\langle g^{-1},[\mathfrak{D}, g], \ldots,\left[\mathfrak{D}, g^{-1}\right],[\mathfrak{D}, g]\right\rangle .
\end{aligned}
$$

Hence the lemma follows.

2.2. Entireness of the b-JLO Chern character. For $A \in{ }^{\mathrm{b}} \Psi^{-\infty}(\widehat{M}, \mathcal{V})$, we let

$$
\operatorname{Tr}^{M}(A):=\int_{M} \operatorname{tr}\left(\left.K_{A}\right|_{\Delta}\right) \text { and }{ }^{\mathrm{b}} \operatorname{Tr}^{\mathrm{end}}(A):=\int_{(-\infty, 0] \times \partial M} \operatorname{tr}\left(\left.K_{A}\right|_{\Delta}\right) .
$$

When $\mathcal{V}$ is a Clifford module of degree 1 , we define

$$
\operatorname{Str}_{(1)}^{M}(A):=\int_{M} \operatorname{str}_{(1)}\left(\left.K_{A}\right|_{\Delta}\right) \quad \text { and } \quad{ }^{\mathrm{b}} \operatorname{Str}_{(1)}^{\mathrm{end}}(A):=\int_{(-\infty, 0] \times \partial M} \operatorname{str}_{(1)}\left(\left.K_{A}\right|_{\Delta}\right) .
$$

When $\left.A\right|_{(-\infty, 0] \times \partial M}$ is of trace class, we also write $\operatorname{Str}_{(1)}^{\mathrm{end}}(A)$ instead of ${ }^{\mathrm{b}} \operatorname{Str}_{(1)}^{\mathrm{end}}(A)$. Now let us give an upper bound in terms of ${ }^{\mathrm{b}}\left\|a_{i}\right\|$ for

$$
\begin{aligned}
\int_{\Delta^{n}}{ }^{\mathrm{b}} \operatorname{Str}_{(1)}\left(a_{0} e^{-\sigma_{0} \mathfrak{D}^{2}}\left[\mathfrak{D}, a_{1}\right] e^{-\sigma_{1} \mathfrak{D}^{2}} \ldots\left[\mathfrak{D}, a_{n}\right] e^{-\sigma_{n} \mathfrak{D}^{2}}\right) d \sigma \\
=\int_{\Delta^{n}} \operatorname{Str}_{(1)}^{M}\left(a_{0} e^{-\sigma_{0} \mathfrak{D}^{2}}\left[\mathfrak{D}, a_{1}\right] e^{-\sigma_{1} \mathfrak{D}^{2}} \ldots\left[\mathfrak{D}, a_{n}\right] e^{-\sigma_{n} \mathfrak{D}^{2}}\right) d \sigma \\
\quad+\int_{\Delta^{n}}{ }^{\mathrm{b}} \operatorname{Str}_{(1)}^{\mathrm{end}}\left(a_{0} e^{-\sigma_{0} \mathfrak{D}^{2}}\left[\mathfrak{D}, a_{1}\right] e^{-\sigma_{1} \mathfrak{D}^{2}} \ldots\left[\mathfrak{D}, a_{n}\right] e^{-\sigma_{n} \mathfrak{D}^{2}}\right) d \sigma
\end{aligned}
$$

For the first summand (2.1), by standard differential calculus on compact manifolds, one has

$\left|\int_{\Delta^{n}} \operatorname{Str}_{(1)}^{M}\left(a_{0} e^{-\sigma_{0} \mathfrak{D}^{2}} \ldots\left[\mathfrak{D}, a_{n}\right] e^{-\sigma_{n} \mathfrak{D}^{2}}\right) d \sigma\right| \leq \operatorname{Tr}^{M}\left(e^{-\mathfrak{D}^{2}}\right){ }^{\mathrm{b}}\left\|a_{0}\right\|^{\mathrm{b}}\left\|a_{1}\right\| \ldots{ }^{\mathrm{b}}\left\|a_{n}\right\|$,

cf. [GS89], Lemma 2.1.

For the second summand (2.2), first notice that on $(-\infty, 0] \times \partial M$,

$$
[\mathfrak{D}, a]=c\left(d a_{c}\right)+e^{x}\left[c\left(a_{\infty} d x\right)+c\left(d a_{\infty}\right)\right]=C+e^{x} B,
$$

where $C=c\left(d a_{c}\right), B=c\left(a_{\infty} d x\right)+c\left(d a_{\infty}\right)$ and $c(-)$ stands for the Clifford multiplication. Similarly, we write

$$
\left[\mathfrak{D}, a_{i}\right]=C_{i}+e^{x} B_{i} \quad \text { and } \quad a_{0}=C_{0}+e^{x} B_{0},
$$

where $C_{i}$ is constant along the normal direction $x$. Notice that $\left\|B_{i}\right\| \leq{ }^{\mathrm{b}}\left\|a_{i}\right\|$ and $\left\|C_{i}\right\| \leq{ }^{\mathrm{b}}\left\|a_{i}\right\|$. Hence the term (2.2) is a sum of the following two types: 
(I) $\int_{\Delta^{n}}{ }^{\mathrm{b}} \operatorname{Str}_{(1)}^{\mathrm{end}}\left(C_{0} e^{-\sigma_{0} \mathfrak{D}^{2}} \ldots C_{n} e^{-\sigma_{n} \mathfrak{D}^{2}}\right) d \sigma$

(II) $\int_{\Delta^{n}}{ }^{\mathrm{b}} \operatorname{Str}_{(1)}^{\mathrm{end}}\left(C_{0} e^{-\sigma_{0} \mathfrak{D}^{2}} \ldots e^{-\sigma_{i} \mathfrak{D}^{2}} e^{x} B_{i} e^{-\sigma_{i+1} \mathfrak{D}^{2}} \ldots C_{n} e^{-\sigma_{n} \mathfrak{D}^{2}}\right) d \sigma$.

Let us denote the Dirac operator on $\mathbb{R} \times \partial M$ by $D_{\mathbb{R}}$ and write $\mathfrak{D}_{\mathbb{R}}=\left(\begin{array}{cc}0 & D_{\mathbb{R}} \\ D_{\mathbb{R}} & 0\end{array}\right)$. By [LMP09], Proposition 3.1, $\left.\left(e^{-\sigma \mathfrak{D}_{\mathbb{R}}^{2}}-e^{-\sigma \mathfrak{D}^{2}}\right)\right|_{(-\infty, 0] \times \partial M}$ is of trace class and there is a constant $\mathcal{K}_{0}$ such that

$$
\left|\operatorname{Tr}\left(e^{-\sigma \mathfrak{D}_{\mathbb{R}}^{2}}-e^{-\sigma \mathfrak{D}^{2}}\right)\right|_{(-\infty, 0] \times \partial M} \mid \leq \mathcal{K}_{0} \quad \text { for all } 0 \leq \sigma \leq 1 .
$$

Type I. Since $\left\|e^{-\sigma \mathfrak{D}_{\mathbb{R}}^{2}}\right\| \leq 1$ and $\left\|e^{-\sigma \mathfrak{D}^{2}}\right\| \leq 1$, one has

$$
\begin{aligned}
& \left|{ }^{\mathrm{b}} \operatorname{Str}_{(1)}^{\mathrm{end}}\left(C_{0} e^{-\sigma_{0} \mathfrak{D}^{2}} \ldots C_{n} e^{-\sigma_{n} \mathfrak{D}^{2}} \mid{ }_{(-\infty, 0] \times \partial M}\right)\right| \\
& \quad \leq(n+1) \mathcal{K}_{0} \prod_{i=0}^{n}\left\|C_{i}\right\|+\left|{ }^{\mathrm{b}} \operatorname{Str}_{(1)}^{\mathrm{end}}\left(\left.C_{0} e^{-\sigma_{0} \mathfrak{D}_{\mathbb{R}}^{2}} \ldots C_{n} e^{-\sigma_{n} \mathfrak{D}_{\mathbb{R}}^{2}}\right|_{(-\infty, 0] \times \partial M}\right)\right| \\
& \quad=(n+1) \mathcal{K}_{0} \prod_{i=0}^{n}\left\|C_{i}\right\|,
\end{aligned}
$$

where the last equality follows from the fact

$$
{ }^{\mathrm{b}} \operatorname{Str}_{(1)}^{\mathrm{end}}\left(\left.C_{0} e^{-\sigma_{0} \mathfrak{D}_{\mathbb{R}}^{2}} \ldots C_{n} e^{-\sigma_{n} \mathfrak{D}_{\mathbb{R}}^{2}}\right|_{(-\infty, 0] \times \partial M}\right)=0
$$

by the definition of the b-trace.

Type II. Due to the presence of the factor $e^{x}$,

$$
C_{0} e^{-\sigma_{0} \mathfrak{D}^{2}} \ldots e^{-\sigma_{i} \mathfrak{D}^{2}} e^{x} B_{i} e^{-\sigma_{i+1} \mathfrak{D}^{2}} \ldots C_{n} e^{-\sigma_{n} \mathfrak{D}^{2}}
$$

is of trace class.

Without loss of generality, it suffices to give an upper bound for

$$
\operatorname{Str}_{(1)}^{\text {end }}\left(e^{x} B_{0} e^{-\sigma_{0} \mathfrak{D}^{2}} A_{1} e^{-\sigma_{1} \mathfrak{D}^{2}} \ldots A_{n} e^{-\sigma_{n} \mathfrak{D}^{2}}\right),
$$

where $A_{i}=B_{i}$ or $C_{i}$ as defined above for $1 \leq i \leq n$. First by the inequality (2.3),

$$
\begin{aligned}
& \left|\operatorname{Str}_{(1)}^{\mathrm{end}}\left(e^{x} B_{0} e^{-\sigma_{0} \mathfrak{D}^{2}} A_{1} e^{-\sigma_{1} \mathfrak{D}^{2}} \ldots A_{n} e^{-\sigma_{n} \mathfrak{D}^{2}}\right)\right| \\
& \leq(n+1) \mathcal{K}_{0}\left\|B_{0}\right\| \prod_{i=1}^{n}\left\|A_{i}\right\| \\
& \quad+\left|\operatorname{Str}_{(1)}^{\text {end }}\left(\left.e^{x} B_{0} e^{-\sigma_{0} \mathfrak{D}_{\mathbb{R}}^{2}} A_{1} e^{-\sigma_{1} \mathfrak{D}_{\mathbb{R}}^{2}} \ldots A_{n} e^{-\sigma_{n} \mathfrak{D}_{\mathbb{R}}^{2}}\right|_{(-\infty, 0] \times \partial M}\right)\right| .
\end{aligned}
$$

Now we can rewrite

$$
\begin{aligned}
& e^{x} B_{0} e^{-\sigma_{0} \mathfrak{D}_{\mathbb{R}}^{2}} A_{1} e^{-\sigma_{1} \mathfrak{D}_{\mathbb{R}}^{2}} \ldots A_{n} e^{-\sigma_{n} \mathfrak{D}_{\mathbb{R}}^{2}} \\
& =\left(e^{x} B_{0} e^{-\sigma_{0} \mathfrak{D}_{\mathbb{R}}^{2}} e^{-\beta_{1} x}\right)\left(e^{\beta_{1} x} A_{1} e^{-\sigma_{1} \mathfrak{D}_{\mathbb{R}}^{2}} e^{-\beta_{2} x}\right) e^{\beta_{2} x} \ldots e^{-\beta_{n} x}\left(e^{\beta_{n} x} A_{n} e^{-\sigma_{n} \mathfrak{D}_{\mathbb{R}}^{2}}\right),
\end{aligned}
$$


with $1>\beta_{1}>\beta_{2}>\cdots>\beta_{n}>0$. By [LMP09], Proposition 3.7, there is a constant $\mathcal{K}^{\prime}$ such that

$$
\left\|e^{\beta_{i} x} A_{i} e^{-\sigma_{i} \mathfrak{D}_{\mathbb{R}}^{2}} e^{-\beta_{i+1} x}\right\|_{\sigma_{i}^{-1}} \leq \mathcal{K}^{\prime}\left\|A_{i}\right\|\left(\beta_{i}-\beta_{i+1}\right)^{-\sigma_{i}}\left(\sigma_{i}^{-\frac{\mathrm{dim} M+1}{2} \sigma_{i}}\right)
$$

for all $i$. Notice that

$$
\sigma^{-\frac{\operatorname{dim} M+1}{2} \sigma}=e^{-\frac{\operatorname{dim} M+1}{2} \sigma \ln (\sigma)}
$$

is bounded on [0,1]. If we take $\beta_{i}=(n+1-i) /(n+1)$, then by the Hölder inequality one has

$$
\begin{gathered}
\left|\operatorname{Str}_{(1)}^{\text {end }}\left(\left.e^{x} B_{0} e^{-\sigma_{0} \mathcal{D}_{\mathbb{R}}^{2}} A_{1} e^{-\sigma_{1} \mathfrak{D}_{\mathbb{R}}^{2}} \ldots A_{n} e^{-\sigma_{n} \mathfrak{D}_{\mathbb{R}}^{2}}\right|_{(-\infty, 0] \times \partial M}\right)\right| \\
\leq \mathcal{K}_{1}^{n+1}(n+1)\left\|B_{0}\right\| \prod_{i=1}^{n}\left\|A_{i}\right\|
\end{gathered}
$$

for some fixed constant $\mathcal{K}_{1}$.

Applying the estimates above, we have the following proposition.

Proposition 2.4. ${ }^{\mathrm{b}} \mathrm{Ch}^{\bullet}(\mathfrak{D})$ is an entire cyclic cochain.

Proof. We have

$$
\begin{aligned}
\left.\right|^{\mathrm{b}} \mathrm{Ch}^{n} & (\mathfrak{D})\left(a_{0}, \ldots, a_{n}\right) \mid \\
& =\left.\right|^{\mathrm{b}}\left\langle a_{0},\left[\mathfrak{D}, a_{1}\right], \ldots,\left[\mathfrak{D}, a_{n}\right]\right\rangle \mid \\
& =\left|\int_{\Delta^{n}}{ }^{\mathrm{b}} \operatorname{Str}_{(1)}\left(a_{0} e^{-\sigma_{0} \mathfrak{D}^{2}}\left[\mathfrak{D}, a_{1}\right] e^{-\sigma_{1} \mathfrak{D}^{2}} \ldots\left[\mathfrak{D}, a_{n}\right] e^{-\sigma_{n} \mathfrak{D}^{2}}\right) d \sigma\right| \\
& \leq \frac{2^{n}(n+1)\left(\mathcal{K}_{1}^{n}+2 \mathcal{K}_{0}\right)}{n !}\left\|a_{0}\right\|^{\mathrm{b}}\left\|a_{1}\right\| \ldots{ }^{\mathrm{b}}\left\|a_{n}\right\| .
\end{aligned}
$$

It follows that ${ }^{\mathrm{b}} \mathrm{Ch}^{\bullet}(\mathfrak{D})$ defines a continuous linear functional on $C_{-}^{\omega}(A)$, i.e., an entire cyclic cochain in $C_{\omega}^{-}(A)$.

\section{Odd APS index theorem for manifolds with boundary}

In this section, we shall state and prove the main theorem of this paper. Let $\hat{M}$ be an odd-dimensional spin b-manifold with a b-metric as before and $D$ its associated Dirac operator. Recall that the spinor bundle $S$ of $\hat{M}$ naturally induces a Clifford module of degree 1 , denoted by $S \otimes \mathbb{C}^{1 \mid 1}$, where the generator $e_{1}$ of $\mathrm{Cl}_{1}(\mathbb{C})$ acts on $S \otimes \mathbb{C}^{1 \mid 1}$ by $\left(\begin{array}{cc}0 & 1 \\ -1 & 0\end{array}\right)$, where $\mathbb{C}^{1 \mid 1}=\mathbb{C}^{+} \oplus \mathbb{C}^{-}$is $\mathbb{Z}_{2}$-graded. We put

$$
\mathfrak{D}=\left(\begin{array}{cc}
0 & D \\
D & 0
\end{array}\right) \quad \text { and } \quad \mathfrak{D}_{t}=t \mathfrak{D}
$$


We define

$$
{ }^{\mathrm{b}} \mathrm{Ch}^{n}(\mathfrak{D}, t)\left(a_{0}, a_{1}, \ldots, a_{n}\right):={ }^{\mathrm{b}}\left\langle\left\langle a_{0},\left[\mathfrak{D}_{t}, a_{1}\right], \ldots,\left[\mathfrak{D}_{t}, a_{n}\right]\right\rangle\right\rangle
$$

for $a_{i} \in C_{\exp }^{\infty}(\widehat{M})$. Here

$$
{ }^{\mathrm{b}}\left\langle\left\langle A_{0}, A_{1}, \ldots, A_{n}\right\rangle\right\rangle:=\int_{\Delta^{n}}{ }^{\mathrm{b}} \operatorname{Str}_{(1)}\left(A_{0} e^{-\sigma_{0}\left(d \mathfrak{D}_{t}+\mathfrak{D}_{t}^{2}\right)} \ldots A_{n} e^{-\sigma_{n}\left(d \mathfrak{D}_{t}+\mathfrak{D}_{t}^{2}\right)}\right) d \sigma,
$$

with

$$
e^{-s\left(d \mathfrak{D}_{t}+\mathfrak{D}_{t}^{2}\right)}:=\sum_{k=0}^{\infty}(-s)^{k} \int_{\Delta^{k}} e^{-\sigma_{0} s \mathfrak{D}_{t}^{2}} d \mathfrak{D}_{t} e^{-\sigma_{1} s \mathfrak{D}_{t}^{2}} \ldots d \mathfrak{D}_{t} e^{-\sigma_{k} s \mathfrak{D}_{t}^{2}} d \sigma .
$$

Notice that (cf. [Get93a], Lemma 2.5)

$$
{ }^{\mathrm{b}}\left\langle\left\langle A_{0}, A_{1}, \ldots, A_{n}\right\rangle\right\rangle={ }^{\mathrm{b}}\left\langle A_{0}, \ldots, A_{n}\right\rangle-\sum_{i=0}^{n}{ }^{\mathrm{b}}\left\langle A_{0}, \ldots, A_{i}, d t \mathfrak{D}, A_{i+1}, \ldots, A_{n}\right\rangle
$$

Therefore

$$
\begin{aligned}
{ }^{\mathrm{b}} \mathrm{Ch}^{k}(\mathfrak{D}, t)\left(a_{0}, \ldots, a_{n}\right) \\
={ }^{\mathrm{b}}\left\langle a_{0},\left[\mathfrak{D}_{t}, a_{1}\right], \ldots,\left[\mathfrak{D}_{t}, a_{n}\right]\right\rangle \\
\quad-\sum_{i=0}^{n}(-1)^{i} d t^{\mathrm{b}}\left\langle a_{0},\left[\mathfrak{D}_{t}, a_{1}\right], \ldots,\left[\mathfrak{D}_{t}, a_{i}\right], \mathfrak{D},\left[\mathfrak{D}_{t}, a_{i+1}\right], \ldots,\left[\mathfrak{D}_{t}, a_{n}\right]\right\rangle .
\end{aligned}
$$

Recall that

$$
{ }^{\mathrm{b}} \operatorname{Tr}[Q, K]=\frac{i}{2 \pi} \int_{-\infty}^{\infty}{ }^{\partial} \operatorname{Tr}\left(\frac{d \mathrm{I}(Q, \lambda)}{d \lambda} \mathrm{I}(K, \lambda)\right) d \lambda
$$

if either $Q$ or $K$ is in $\Psi_{b}^{-\infty}(\hat{M}, \mathcal{V})$, where $\mathrm{I}(Q, \lambda)$ (resp. $\left.\mathrm{I}(K, \lambda)\right)$ is the indicial family of $Q$ (resp. $K$ ), cf. [Loy05], Theorem 2.5. For the Dirac operator $D$ above, we have

$$
I(D, \lambda)={ }^{\partial} D+i \lambda c(v),
$$

where $v=d x$ is the normal cotangent vector and $c(v)$ is the Clifford multiplication of $v$, cf. [Get93b], Proposition 5.4. In the following identities ([Get93a], Lemma 6.3), we assume that the indicial family $I\left(A_{i}, \lambda\right)$ of $A_{i}$ is independent of $\lambda$ and commutes with $\mathrm{Cl}_{1}(\mathbb{C})$ and $c(v)$. Let us denote the degree of $A_{i}$ with respect to the $\mathbb{Z}_{2}$-grading by $\left|A_{i}\right|$.

(1) If we denote $\varepsilon_{i}=\left(\left|A_{0}\right|+\cdots+\left|A_{i-1}\right|\right)\left(\left|A_{i}\right|+\cdots+\left|A_{n}\right|\right)$, then

$$
\begin{array}{r}
{ }^{\mathrm{b}}\left\langle\left\langle A_{0}, A_{1}, \ldots, A_{n}\right\rangle\right\rangle=(-1)^{\varepsilon_{i} \mathrm{~b}}\left\langle\left\langle A_{i}, \ldots, A_{n}, A_{0}, \ldots, A_{i-1}\right\rangle\right\rangle . \\
\text { (2) } \sum_{i=0}^{n}(-1)^{\varepsilon_{i} \mathrm{~b}}\left\langle\left\langle 1, A_{i}, \ldots, A_{n}, A_{0}, \ldots, A_{i-1}\right\rangle\right\rangle={ }^{\mathrm{b}}\left\langle\left\langle A_{0}, A_{1}, \ldots, A_{n}\right\rangle\right\rangle .
\end{array}
$$


(3) We have

$$
\begin{aligned}
& { }^{\mathrm{b}}\left\langle\left\langle A_{0}, \ldots, A_{i-1},\left[d \mathfrak{D}_{t}+\mathfrak{D}_{t}^{2}, A_{i}\right], A_{i+1}, \ldots, A_{n}\right\rangle\right\rangle \\
& \quad={ }^{\mathrm{b}}\left\langle\left\langle A_{0}, \ldots, A_{i-1} A_{i}, \ldots, A_{n}\right\rangle\right\rangle-{ }^{\mathrm{b}}\left\langle\left\langle A_{0}, \ldots, A_{i} A_{i+1}, \ldots, A_{n}\right\rangle\right\rangle .
\end{aligned}
$$

(4) Write $\delta_{i}=\left|A_{0}\right|+\cdots+\left|A_{i-1}\right|$. Then

$$
\begin{aligned}
& d^{\mathrm{b}}\left\langle\left\langle A_{0}, A_{1}, \ldots, A_{n}\right\rangle\right\rangle-\left\langle\left\langle{ }^{\partial} A_{0},{ }^{\partial} A_{1}, \ldots,{ }^{\partial} A_{n}\right\rangle\right\rangle \\
& \quad=\sum_{i=0}^{n}(-1)^{\delta_{i} \mathrm{~b}}\left\langle\left\langle A_{0}, A_{1}, \ldots, A_{i-1},\left[d+\mathfrak{D}_{t}, A_{i}\right], A_{i+1}, \ldots, A_{n}\right\rangle\right\rangle .
\end{aligned}
$$

Here we define

$$
\begin{aligned}
{ }^{\partial}\left\langle\left\langle{ }^{\partial} A_{0},{ }^{\partial} A_{1}, \ldots,{ }^{\partial} A_{n}\right\rangle\right\rangle \\
\quad=\int_{\Delta^{n}}{ }^{\mathrm{b}} \operatorname{Str}_{(2)}\left({ }^{\partial} A_{0} e^{-\sigma_{0}\left(d^{\partial} \mathfrak{D}_{t}+{ }^{\partial} \mathfrak{D}_{t}^{2}\right)} \ldots{ }^{\partial} A_{n} e^{-\sigma_{n}\left(d^{\partial} \mathfrak{D}_{t}+{ }^{\partial} \mathfrak{D}_{t}^{2}\right)}\right) d \sigma,
\end{aligned}
$$

where

$$
{ }^{\mathrm{b}} \operatorname{Str}_{(2)}(B)=\frac{1}{4 \pi}{ }^{\mathrm{b}} \operatorname{Str}\left(e_{1} e_{2} B\right) \quad \text { with } e_{1}=\left(\begin{array}{cc}
0 & 1 \\
-1 & 0
\end{array}\right), e_{2}=\left(\begin{array}{cc}
0 & c(v) \\
c(v) & 0
\end{array}\right)
$$

and

$$
{ }^{\partial} \mathfrak{D}_{t}=t^{\partial} \mathfrak{D} \quad \text { with }{ }^{\partial} \mathfrak{D}=\left(\begin{array}{cc}
0 & { }^{\partial} D \\
{ }^{\partial} D & 0
\end{array}\right)
$$

Now if we let $A_{0}=a_{0}$ and $A_{i}=\left[\mathfrak{D}_{t}, a_{i}\right]$ for $1 \leq i \leq n$, where $a_{i} \in C_{\exp }^{\infty}(\widehat{M})$, then it is easy to see that

$$
\left[d+\mathfrak{D}_{t}, A_{0}\right]=\left[d+\mathfrak{D}_{t}, a_{0}\right]=\left[\mathfrak{D}_{t}, a_{0}\right]
$$

and

$$
\left[d+\mathfrak{D}_{t}, A_{i}\right]=\left[d+\mathfrak{D}_{t},\left[\mathfrak{D}_{t}, a_{i}\right]\right]=\left[d \mathfrak{D}_{t}+\mathfrak{D}_{t}^{2}, a_{i}\right] \quad \text { with } 1 \leq i \leq n .
$$

Therefore, by applying the identities above to $A_{0}=a_{0}$ and $A_{i}=\left[\mathfrak{D}_{t}, a_{i}\right]$, we see that ([Get93a], Theorem 6.2)

$$
(d-b-B){ }^{\mathrm{b}} \mathrm{Ch}^{\bullet}(\mathfrak{D}, t)=\mathrm{Ch}^{\bullet}\left({ }^{\partial} \mathfrak{D}, t\right),
$$

where

$$
\mathrm{Ch}^{n}\left({ }^{\partial} \mathfrak{D}, t\right)\left({ }^{\partial} a_{0},{ }^{\partial} a_{1}, \ldots,{ }^{\partial} a_{n}\right)={ }^{\partial}\left\langle\left\langle{ }^{\partial} a_{0},\left[{ }^{\partial} \mathfrak{D}_{t},{ }^{\partial} a_{1}\right], \ldots,\left[{ }^{\partial} \mathfrak{D}_{t},{ }^{\partial} a_{n}\right]\right\rangle\right\rangle .
$$

Let $\alpha \in \Omega^{*}(0, \infty)$ be the differential form $\alpha=\left\langle{ }^{\mathrm{b}} \mathrm{Ch}^{\bullet}(\mathfrak{D}, t), \mathrm{Ch}_{\bullet}(g)\right\rangle$. Then

$$
d \alpha=\left\langle\mathrm{Ch}^{\bullet}\left({ }^{\partial_{\mathfrak{D}}, t}\right), \mathrm{Ch}_{\bullet}\left({ }^{\partial} g\right)\right\rangle .
$$


By the fundamental theorem of calculus,

$$
\alpha\left(t_{2}\right)-\alpha\left(t_{1}\right)=\left\langle\int_{t_{1}}^{t_{2}} \mathrm{Ch}^{\bullet}\left({ }^{\partial} \mathfrak{D}, t\right), \mathrm{Ch}_{\bullet}\left({ }^{\partial} g\right)\right\rangle .
$$

Therefore, if both of the following limits exist, then

$$
\lim _{t \rightarrow \infty} \alpha(t)-\lim _{t \rightarrow 0} \alpha(t)=-\left\langle\eta^{\bullet}\left({ }^{\partial} \mathfrak{D}\right), C_{\bullet}\left({ }^{\partial} g\right)\right\rangle \text {. }
$$

Here $\eta^{\bullet}\left({ }^{\partial} \mathfrak{D}\right)=\int_{0}^{\infty} \mathrm{Ch}^{\bullet}\left({ }^{\partial} \mathfrak{D}, t\right)$, which is the higher eta cochain of Wu [Wu93], although our normalization factor is different from that of Wu. More explicitly,

$$
\begin{aligned}
\eta^{2 k+1}\left({ }^{\partial} \mathfrak{D}\right)\left(a_{0}, a_{1}, \ldots, a_{2 k+1}\right)= & \frac{1}{2 \pi} \sum_{i=0}^{2 k+1}(-1)^{i} \int_{0}^{\infty} d t^{\partial}\left\langle a_{0},\left[{ }^{\partial} D_{t}, a_{1}\right], \ldots\right. \\
& \left.\ldots,\left[{ }^{\partial} D_{t}, a_{i}\right],{ }^{\partial} D,\left[{ }^{\partial} D_{t}, a_{i+1}\right], \ldots,\left[{ }^{\partial} D_{t}, a_{2 k+1}\right]\right\rangle_{t}
\end{aligned}
$$

where

$$
{ }^{\partial}\left\langle A_{0}, \ldots, A_{n}\right\rangle_{t}=\int_{\Delta^{n}} \operatorname{Str}\left(A_{0} e^{-\sigma_{0}{ }^{\partial} D_{t}^{2}} \ldots A_{n} e^{-\sigma_{n}{ }^{\partial} D_{t}^{2}}\right) d \sigma .
$$

Let us also write $\eta^{\bullet}\left({ }^{\partial} D\right)=\eta^{\bullet}\left({ }^{\partial} \mathfrak{D}\right)$. This higher eta cochain $\eta^{\bullet}\left({ }^{\partial} D\right)$ has a finite radius of convergence, cf. [Wu93], Proposition 1.5. In order for

$$
\left\langle\eta^{\bullet}\left({ }^{\partial} D\right), \mathrm{Ch}_{\bullet}\left({ }^{\partial} g\right)\right\rangle
$$

to converge, we shall make the following assumptions throughout the rest of this paper:

(1) ${ }^{\partial} D$ is invertible and the lowest eigenvalue of $\left|{ }^{\partial} D\right|$ is $\lambda$;

(2) $\left\|\left[{ }^{\partial} D,{ }^{\partial} g\right]\right\|=\left\|d{ }^{\partial} g\right\|<\lambda$.

Let us denote by

$$
\mathrm{Ch}_{\bullet}^{\mathrm{dR}}(g):=\sum_{k=0}^{\infty} \frac{1}{(2 \pi i)^{k+1}} \frac{k !}{(2 k+1) !} \operatorname{tr}\left(\left(g^{-1} d g\right)^{2 k+1}\right) \in \Omega^{*}(\hat{M})
$$

the Chern character of $g$ in the de Rham cohomology of $\widehat{M}$. Then we have the following main theorem of this paper.

Theorem 3.1. Let $\widehat{M}$ be an odd-dimensional spin $b$-manifold with a b-metric and $D$ its associated Dirac operator. Assume that ${ }^{\partial} D$ is invertible. Let $g \in U_{k}\left(C^{\infty}(\widehat{M})\right)$ be a unitary over $\hat{M}$. If $\left\|d^{\partial} g\right\|<\lambda$, where $\lambda$ is the lowest nonzero eigenvalue of $\left|{ }^{\partial} D\right|$, then

$$
\operatorname{sf}\left(D, g^{-1} D g\right)=f_{\widehat{M}} \hat{A}(\hat{M}) \wedge \mathrm{Ch}_{\bullet}^{\mathrm{dR}}(g)-\left\langle\eta^{\bullet}\left({ }^{\partial} D\right), \mathrm{Ch}_{\bullet}\left({ }^{\partial} g\right)\right\rangle .
$$

Here $\hat{A}(\widehat{M})=\operatorname{det}\left(\frac{{ }^{\mathrm{b}} \nabla^{2} / 4 \pi i}{\sinh ^{\mathrm{b}} \nabla^{2} / 4 \pi i}\right)^{1 / 2}$, with ${ }^{\mathrm{b}} \nabla$ the Levi-Civita b-connection associated to the $b$-metric on $\hat{M}$. 
Proof. We need to identify the limits of $\alpha(t)$ for $t=\infty$ and $t=0$. In the case of closed manifolds, the local formula for the limit of $\alpha(t)$ as $t \rightarrow 0$ follows from Getzler's asymptotic calculus, cf. [BF90], [CM90], [Get83]. A direct calculation in the b-calculus setting is carried out in [LMP09], Sections 5 and 6. In particular, we have

$$
\lim _{t \rightarrow 0} \alpha(t)=\lim _{t \rightarrow 0}\left\langle{ }^{\mathrm{b}} \mathrm{Ch} \cdot(\mathfrak{D}), \mathrm{Ch}_{\bullet}(g)\right\rangle=f_{\widehat{M}} \hat{A}(\hat{M}) \wedge \mathrm{Ch}_{\bullet}^{\mathrm{dR}}(g) .
$$

Now the theorem follows immediately once we have

$$
\lim _{t \rightarrow \infty} \alpha(t)=\operatorname{sf}\left(D, g^{-1} D g\right),
$$

which we will prove in Proposition 5.8 below.

Corollary 3.2. With the same notation as above,

$$
\left\langle\eta^{\bullet}\left({ }^{\partial} D\right), C_{\bullet}\left({ }^{\partial} g\right)\right\rangle=-\bar{\eta}\left(\partial \hat{M},{ }^{\partial} g\right) \bmod \mathbb{Z} .
$$

Proof. Here $\bar{\eta}\left(\partial \hat{M},{ }^{g}\right)$ is the eta invariant of Dai and Zhang [DZ06]. Without loss of generality, we can assume that the unitary $g$ is constant along the normal direction of the cylindrical end. In this case, we have

$$
f_{\widehat{M}} \hat{A}(\hat{M}) \wedge \mathrm{Ch}_{\bullet}^{\mathrm{dR}}(g)=\int_{M} \hat{A}(M) \wedge \mathrm{Ch}_{\bullet}^{\mathrm{dR}}(g)
$$

by definition of the regularized integral. Now comparing the above theorem with the Toeplitz index theorem on odd-dimensional manifolds by Dai and Zhang [DZ06], Theorem 2.3, we have

$$
\left\langle\eta^{\bullet}\left({ }^{\partial} D\right), \text { Ch. }\left({ }^{\partial} g\right)\right\rangle=-\bar{\eta}\left(\partial \hat{M},{ }^{\partial} g\right) \bmod \mathbb{Z} .
$$

This equality provides more evidence for the naturality of the Dai-Zhang eta invariant for even-dimensional closed manifolds.

\section{Spectral flow}

In this section, we proceed to explain the notion of spectral flow and prove an analogue of Getzler's formula for spectral flow (cf. [Get93b], Corollary 2.7) in the b-calculus setting.

Following Booss-Bavnbek, Lesch and Phillips [BBLP05], we define the notion of spectral flow as follows.

Definition 4.1. Let $T_{u}: \mathscr{H} \rightarrow \mathscr{H}$ for $u \in[0,1]$ be a continuous path of (possibly unbounded) self-adjoint Fredholm operators. Then its spectral flow, denoted by $\operatorname{sf}\left(T_{u}\right)_{0 \leq u \leq 1}$, is defined by

$$
\operatorname{sf}\left(T_{u}\right)_{0 \leq u \leq 1}=\operatorname{wind}\left(\kappa\left(T_{u}\right)_{0 \leq u \leq 1}\right),
$$


where $\kappa(T)=(T-i)(T+i)^{-1}$ is the Cayley transform of $T$ and wind $\left(\kappa\left(T_{u}\right)_{0 \leq u \leq 1}\right)$ is the winding number of the path $\kappa\left(T_{u}\right)_{0 \leq u \leq 1}$ (see also [KL04], Section 6). We also write $\operatorname{sf}\left(T_{0}, T_{1}\right)$ for the spectral flow if it is clear what the path is from the context.

Actually, in this paper, where we are concerned with smooth paths of self-adjoint Fredholm operators, we use the following equivalent working definition of the spectral flow (cf. [BBLP05, Section 2.2]). Let $T_{u}: \mathscr{H} \rightarrow \mathscr{H}$ for $u \in[0,1]$ be a smooth path of (possibly unbounded) self-adjoint Fredholm operators. For a fixed $u_{0} \in[0,1]$, there exists $(a, b) \subset[0,1]$ such that

(1) $u_{0} \in(a, b)$ (unless $u_{0}=0$ or 1 , in which case $u_{0}=a=0$ if $u_{0}=0$, and $u_{0}=b=1$ if $\left.u_{0}=1\right)$;

(2) $\operatorname{dim} \operatorname{ker}\left(T_{u}\right) \leq \operatorname{dim} \operatorname{ker}\left(T_{u_{0}}\right)$ for all $u \in(a, b)$.

By shrinking the neighborhood $(a, b)$ if necessary, we can assume that the essential spectrum of $\left|T_{u}\right|$ for $u \in(a, b)$ is bounded below uniformly by $\lambda_{0}$ and the spectrum of $T_{u}$ in $\left(-\lambda_{0}, \lambda_{0}\right)$ consists of discrete eigenvalues. We can further assume $T_{u}$ has the same number of eigenvalues (counted with multiplicities) in $\left(-\lambda_{0}, \lambda_{0}\right)$, for all $u \in(a, b)$. By perturbation theory of linear operators (cf. [Kat95], II.6, V.4.3, VII.3), there are smooth functions $\beta_{k}$ on $(a, b)$ such that $\left\{\beta_{k}(u)\right\}_{k}$ gives a complete set of eigenvalues of $T_{u}$ in $\left(-\lambda_{0}, \lambda_{0}\right)$. Let $n_{b}$ (resp. $n_{a}$ ) be the number of nonnegative eigenvalues of $T_{b}$ (resp. $\left.T_{a}\right)$ in $\left(-\lambda_{0}, \lambda\right)$. Then we define the spectral flow of $\left(T_{u}\right)_{a \leq u \leq b}$ to be

$$
\operatorname{sf}\left(T_{u}\right)_{a \leq u \leq b}:=\left(n_{b}-n_{a}\right) .
$$

We call an interval $(a, b)$ as above together with $u_{0} \in(a, b)$ a pointed gap interval. It is easy to see that the formula (4.1) is additive with respect to disjoint pointed gap intervals. Let us cover $[0,1]$ by finitely many intervals, say $\left[a_{i}, b_{i}\right]_{0 \leq i \leq n}$ such that each $\left(a_{i}, b_{i}\right)$ is a pointed gap interval, with $b_{i}=a_{i+1}, u_{0}=a_{0}=0, u_{n}=b_{n}=1$ and $u_{j} \in\left(a_{j}, b_{j}\right)$ for $1 \leq j \leq n-1$. Then we define

$$
\operatorname{sf}\left(T_{u}\right)_{0 \leq u \leq 1}:=\sum_{j=0}^{n} \operatorname{sf}\left(T_{u}\right)_{a_{j} \leq u \leq b_{j}} .
$$

By additivity of formula (4.1), we see that $\operatorname{sf}\left(T_{u}\right)_{0 \leq u \leq 1}$ so defined is independent of the choice of pointed gap intervals.

Let $\widehat{M}$ be an odd-dimensional spin b-manifold with a b-metric as before and $D$ its associated Dirac operator. Let $D_{u}=(1-u) D+u g^{-1} D g$. Since by assumption $\left\|\left[{ }^{\partial} D,{ }^{\partial} g\right]\right\|<\lambda$, we see that ${ }^{\partial} D_{u}$ is invertible for all $u \in[0,1]$. It follows that inf $\operatorname{spec}_{\text {ess }}\left(\left|D_{u}\right|\right)>0$ for all $u \in[0,1]$. Thus $\left\{D_{u}\right\}_{0 \leq u \leq 1}$ is an analytic family of self-adjoint Fredholm operators.

Following from the discussion above, we see that for each fixed $u_{0} \in[0,1]$, there exists $(a, b) \subset[0,1]$ and $\lambda_{0}>0$ such that the spectrum of $D_{u}$ in $\left(-\lambda_{0}, \lambda_{0}\right)$ consists of discrete eigenvalues for all $u \in(a, b)$. Moreover, we can assume $D_{u}$ has the same 
number of eigenvalues in $\left(-\lambda_{0}, \lambda_{0}\right)$ for all $u \in(a, b)$. We put

$$
\begin{aligned}
& A_{u}:=D_{u} P_{u}, \\
& B_{u}:=D_{u}\left(I-P_{u}\right)+P_{u}, \\
& C_{u}:=D_{u}\left(I-P_{u}\right),
\end{aligned}
$$

where $P_{u}$ is the spectral projection of $D_{u}$ on $\left(-\lambda_{0}, \lambda_{0}\right)$. Let $\beta_{k}$ be the smooth functions on $(a, b)$ such that $\left\{\beta_{k}(u)\right\}_{k}$ gives the complete set of eigenvalues (counted with multiplicities) of $A_{u}$. Since $\left\{D_{u}\right\}_{0 \leq u \leq 1}$ is an analytic family of operators, $\beta_{k}$ is an analytic function of $u \in(a, b)$. It follows that for each $k, \beta_{k}$ either has only finitely many isolated zeroes or is itself constantly zero. Hence by shrinking $(a, b)$ as much as needed, we can assume that $\beta_{k}$ either is a constant zero function or has only one zero in $(a, b)$. In the latter case, by shrinking $(a, b)$ again if necessary, we can assume that the isolated zeros can only occur at $u_{0}$. Moreover, for each $u \in(a, b)$, there is a set of orthonormal eigenvectors $\left\{\phi_{k}(u)\right\}_{1 \leq k \leq m}$ such that $A_{u} \phi_{k}(u)=\beta_{k}(u) \phi_{k}(u)$ and the vector-valued function $\phi_{k}$ is analytic with respect to $u$ for each $1 \leq k \leq m$.

Following Getzler [Get93b], we define the truncated eta invariant of $D$ to be

$$
\eta_{\varepsilon}(D):=\frac{1}{\sqrt{\pi}} \int_{\varepsilon}^{\infty}{ }^{\mathrm{b}} \operatorname{Tr}\left(D e^{-s D^{2}}\right) s^{-1 / 2} d s=\frac{2}{\sqrt{\pi}} \int_{\varepsilon}^{\infty}{ }^{\mathrm{b}} \operatorname{Tr}\left(D e^{-t^{2} D^{2}}\right) d t
$$

and the reduced (truncated) eta invariant of $D$ to be

$$
\xi_{\varepsilon}(D)=\frac{\eta_{\varepsilon}(D)+\operatorname{dim} \operatorname{ker}(D)}{2} .
$$

The following lemma is a natural extension of [Get93b], Proposition 2.5, to the bcalculus setting.

Lemma 4.2. We have

$$
\frac{d \eta_{\varepsilon}\left(B_{u}\right)}{d u}=-\frac{2 \varepsilon}{\sqrt{\pi}} \mathrm{b} \operatorname{Tr}\left(\dot{B}_{u} e^{-\varepsilon^{2} B_{u}^{2}}\right)+E_{\varepsilon}(u),
$$

where $E_{\varepsilon}(u)$ is defined by

$$
\begin{aligned}
E_{\varepsilon}(u)= & -\frac{2}{\sqrt{\pi}} \int_{\varepsilon}^{\infty} \int_{0}^{1} t^{2 \mathrm{~b}} \operatorname{Tr}\left[e^{-s t^{2} B_{u}^{2}} B_{u}^{2}, \dot{B}_{u} e^{-(1-s) t^{2} B_{u}^{2}}\right] d s d t \\
& -\frac{2}{\sqrt{\pi}} \int_{\varepsilon}^{\infty} \int_{0}^{1} t^{2 \mathrm{~b}} \operatorname{Tr}\left[e^{-s t^{2} B_{u}^{2}} B_{u}, \dot{B}_{u} B_{u} e^{-(1-s) t^{2} B_{u}^{2}}\right] d s d t
\end{aligned}
$$


Proof. Using Duhamel's principle, we have

$$
\begin{aligned}
& \frac{d}{d u} \eta_{\varepsilon}\left(B_{u}\right) \\
&=\frac{2}{\sqrt{\pi}} \int_{\varepsilon}^{\infty}{ }^{\mathrm{b}} \operatorname{Tr}\left(\dot{B}_{u} e^{-t^{2} B_{u}^{2}}\right) d t \\
&-\frac{2}{\sqrt{\pi}} \int_{\varepsilon}^{\infty} \int_{0}^{1}{ }^{\mathrm{b}} \operatorname{Tr}\left(B_{u} e^{-s t^{2} B_{u}^{2}} t^{2}\left(B_{u} \dot{B}_{u}+\dot{B}_{u} B_{u}\right) e^{-(1-s) t^{2} B_{u}^{2}}\right) d s d t \\
&= \frac{2}{\sqrt{\pi}} \int_{\varepsilon}^{\infty}{ }^{\mathrm{b}} \operatorname{Tr}\left(\dot{B}_{u} e^{-t^{2} B_{u}^{2}}\right) d t-\frac{4}{\sqrt{\pi}} \int_{\varepsilon}^{\infty}{ }^{\mathrm{b}} \operatorname{Tr}\left(t^{2} \dot{B}_{u} B_{u}^{2} e^{-t^{2} B_{u}^{2}}\right) d t+E_{\varepsilon}(u) .
\end{aligned}
$$

Integration by parts shows that

$$
\begin{aligned}
\int_{\varepsilon}^{\infty}{ }^{\mathrm{b}} \operatorname{Tr}\left(t^{2} \dot{B}_{u} B_{u}^{2} e^{-t^{2} B_{u}^{2}}\right) d t \\
=-\frac{1}{2} \int_{\varepsilon}^{\infty} t \frac{d}{d t}{ }^{\mathrm{b}} \operatorname{Tr}\left(\dot{B}_{u} e^{-t^{2} B_{u}^{2}}\right) d t \\
=-\left.\frac{1}{2}{ }^{\mathrm{b}} \operatorname{Tr}\left(\dot{B}_{u} e^{-t^{2} B_{u}^{2}}\right) t\right|_{t=\varepsilon} ^{t=\infty}+\frac{1}{2} \int_{\varepsilon}^{\infty}{ }^{\mathrm{b}} \operatorname{Tr}\left(\dot{B}_{u} e^{-t^{2} B_{u}^{2}}\right) d t
\end{aligned}
$$

Since $B_{u}$ is invertible, ${ }^{\mathrm{b}} \operatorname{Tr}\left(t \dot{B}_{u} e^{-t^{2} B_{u}^{2}}\right)$ goes to 0 as $t \rightarrow \infty$. It follows that

$$
\frac{d}{d u} \eta_{\varepsilon}\left(B_{u}\right)=-\frac{2 \varepsilon}{\sqrt{\pi}}^{\mathrm{b}} \operatorname{Tr}\left(\dot{B}_{u} e^{-\varepsilon^{2} B_{u}^{2}}\right)+E_{\varepsilon}(u) .
$$

Corollary 4.3. For $u \in(a, b)$,

$$
\frac{d \eta_{\varepsilon}\left(C_{u}\right)}{d u}=-\frac{2 \varepsilon}{\sqrt{\pi}}{ }^{\mathrm{b}} \operatorname{Tr}\left(\dot{C}_{u} e^{-\varepsilon^{2} C_{u}^{2}}\right)+E_{\varepsilon}(u)
$$

Proof. By definition, we have

$$
\eta_{\varepsilon}\left(C_{u}\right)=\eta_{\varepsilon}\left(B_{u}\right)-K \int_{\varepsilon}^{\infty} e^{-t^{2}} d t
$$

where $K=\operatorname{rank}\left(P_{u}\right)$ is independent of $u \in(a, b)$. Thus $\frac{d}{d u} \eta_{\varepsilon}\left(C_{u}\right)=\frac{d}{d u} \eta_{\varepsilon}\left(B_{u}\right)$. Notice that

$$
\begin{aligned}
& { }^{\mathrm{b}} \operatorname{Tr}\left(\dot{B}_{u} e^{-\varepsilon^{2} B_{u}^{2}}\right) \\
& ={ }^{\mathrm{b}} \operatorname{Tr}\left(\dot{C}_{u} e^{-\varepsilon^{2} C_{u}^{2}}\right)+{ }^{\mathrm{b}} \operatorname{Tr}\left(\dot{P}_{u} e^{-\varepsilon^{2} P_{u}^{2}}\right)+{ }^{\mathrm{b}} \operatorname{Tr}\left(\dot{C}_{u} e^{-\varepsilon^{2} P_{u}^{2}}\right)+{ }^{\mathrm{b}} \operatorname{Tr}\left(\dot{P}_{u} e^{-\varepsilon^{2} C_{u}^{2}}\right) \\
& ={ }^{\mathrm{b}} \operatorname{Tr}\left(\dot{C}_{u} e^{-\varepsilon^{2} C_{u}^{2}}\right)+\operatorname{Tr}\left(\dot{P}_{u} e^{-\varepsilon^{2} P_{u}^{2}}\right)+\operatorname{Tr}\left(\dot{C}_{u} e^{-\varepsilon^{2} P_{u}^{2}}\right)+\operatorname{Tr}\left(\dot{P}_{u} e^{-\varepsilon^{2} C_{u}^{2}}\right)
\end{aligned}
$$


since $\dot{P}_{u} e^{-\varepsilon^{2} P_{u}^{2}}, \dot{C}_{u} e^{-\varepsilon^{2} P_{u}^{2}}$ and $\dot{P}_{u} e^{-\varepsilon^{2} C_{u}^{2}}$ are all trace class operators. In fact, since $P_{u}$ is a projection and the rank of $P_{u}$ remains constant for each $u \in(a, b)$, using Duhamel's formula we have

$$
\operatorname{Tr}\left(\dot{P}_{u} e^{-\varepsilon^{2} P_{u}^{2}}\right)=\frac{1}{2} \operatorname{Tr}\left(\left(\dot{P}_{u} P_{u}+P_{u} \dot{P}_{u}\right) e^{-\varepsilon^{2} P_{u}^{2}}\right)=\frac{-1}{2 \varepsilon^{2}} \frac{d}{d u} \operatorname{Tr}\left(e^{-\varepsilon^{2} P_{u}^{2}}\right)=0 .
$$

By the very definition of $C_{u}$ (see formula (4.2) above), we have $P_{u} C_{u}=C_{u} P_{u}=0$. In particular, $\operatorname{Tr}\left(C_{u} e^{-\varepsilon^{2} P_{u}^{2}}\right) \equiv 0$. Therefore,

$$
\begin{aligned}
0=\frac{d}{d u} \operatorname{Tr}\left(C_{u} e^{-\varepsilon^{2} P_{u}^{2}}\right) & =\operatorname{Tr}\left(\dot{C}_{u} e^{-\varepsilon^{2} P_{u}^{2}}\right)+\operatorname{Tr}\left(C_{u} e^{-\varepsilon^{2} P_{u}^{2}}\left(P_{u} \dot{P}_{u}+\dot{P}_{u} P_{u}\right)\right) \\
& =\operatorname{Tr}\left(\dot{C}_{u} e^{-\varepsilon^{2} P_{u}^{2}}\right) .
\end{aligned}
$$

Similarly, $\operatorname{Tr}\left(\dot{P}_{u} e^{-\varepsilon^{2} C_{u}^{2}}\right)=0$. We conclude that

$$
{ }^{\mathrm{b}} \operatorname{Tr}\left(\dot{B}_{u} e^{-\varepsilon^{2} B_{u}^{2}}\right)={ }^{\mathrm{b}} \operatorname{Tr}\left(\dot{C}_{u} e^{-\varepsilon^{2} C_{u}^{2}}\right) .
$$

Hence the corollary follows.

Lemma 4.4. For $\tau \in(a, b)$ and $\tau \neq u_{0}$, we have

$$
\left.\frac{d}{d u} \eta_{\varepsilon}\left(A_{u}\right)\right|_{u=\tau}=-\left.\frac{2 \varepsilon}{\sqrt{\pi}} \operatorname{Tr}\left(\dot{A}_{u} e^{-\varepsilon^{2} A_{u}^{2}}\right)\right|_{u=\tau} .
$$

Proof. Notice that

$$
\begin{aligned}
\eta_{\varepsilon}\left(A_{u}\right) & =\sum_{k} \frac{2}{\sqrt{\pi}} \int_{\varepsilon}^{\infty} \beta_{k}(u) e^{-t^{2} \beta_{k}^{2}(u)} d t \\
& =\sum_{k} \frac{2}{\sqrt{\pi}} \int_{\varepsilon}^{\infty}\left\langle A_{u} e^{-t^{2} A_{u}^{2}} \phi_{k}(u), \phi_{k}(u)\right\rangle d t .
\end{aligned}
$$

If $\beta_{k}(\tau) \neq 0$, then the same argument from Lemma 4.2 shows that

$$
\left.\frac{d}{d u} \int_{\varepsilon}^{\infty}\left\langle A_{u} e^{-t^{2} A_{u}^{2}} \phi_{k}(u), \phi_{k}(u)\right\rangle d t\right|_{u=\tau}=\left.\varepsilon\left\langle\dot{A_{u}} e^{-\varepsilon^{2} A_{u}^{2}} \phi_{k}(u), \phi_{k}(u)\right\rangle\right|_{u=\tau} .
$$

If $\beta_{k}(\tau)=0$, then $\beta_{k} \equiv 0$ on $(a, b)$ by our choice of the interval $(a, b)$. In particular, $A_{u} \phi_{k}(u)=0$ for all $u \in(a, b)$. Then

$$
\begin{aligned}
\frac{d}{d u}\left\langle A_{u}\right. & \left.\phi_{k}(u), \phi_{k}(u)\right\rangle \\
& =\left\langle\dot{A}_{u} \phi_{k}(u), \phi_{k}(u)\right\rangle+\left\langle A_{u} \dot{\phi}_{k}(u), \phi_{k}(u)\right\rangle+\left\langle A_{u} \phi_{k}(u), \dot{\phi}_{k}(u)\right\rangle \\
& =\left\langle\dot{A}_{u} \phi_{k}(u), \phi_{k}(u)\right\rangle .
\end{aligned}
$$


It follows that $\left\langle\dot{A}_{u} \phi_{k}(u), \phi_{k}(u)\right\rangle=0$. Hence

$$
\left\langle\dot{A}_{u} e^{-\varepsilon^{2} A_{u}^{2}} \phi_{k}(u), \phi_{k}(u)\right\rangle=\left\langle\dot{A}_{u} \phi_{k}(u), \phi_{k}(u)\right\rangle=0
$$

for all $u \in(a, b)$. This finishes the proof.

Corollary 4.5. For $u \in(a, b)$ and $u \neq u_{0}$,

$$
\frac{d}{d u} \eta_{\varepsilon}\left(D_{u}\right) d u=-\frac{2 \varepsilon}{\sqrt{\pi}}^{\mathrm{b}} \operatorname{Tr}\left(\dot{D}_{u} e^{-\varepsilon^{2} D_{u}^{2}}\right)+E_{\varepsilon}(u) .
$$

Proof. Notice that

$$
\eta_{\varepsilon}\left(D_{u}\right)=\eta_{\varepsilon}\left(A_{u}\right)+\eta_{\varepsilon}\left(C_{u}\right)
$$

and

$$
{ }^{\mathrm{b}} \operatorname{Tr}\left(\dot{A}_{u} e^{-\varepsilon^{2} C_{u}^{2}}\right)={ }^{\mathrm{b}} \operatorname{Tr}\left(\dot{C}_{u} e^{-\varepsilon^{2} A_{u}^{2}}\right)=0 .
$$

The corollary follows from the above lemmas.

If we denote by $Q^{+}$the cardinality of the set $\left\{\beta_{k} \mid \beta_{k}\left(u_{0}\right)=0\right.$ and $\left.\beta_{k}(a)>0\right\}$ and by $Q^{-}$the cardinality of the set $\left\{\beta_{k} \mid \beta_{k}\left(u_{0}\right)=0\right.$ and $\left.\beta_{k}(a)<0\right\}$, then

$$
\operatorname{dim} \operatorname{ker} D_{u_{0}}=\operatorname{dim} \operatorname{ker} D_{u}+Q^{+}+Q^{-} \text {for } u \in\left(a, u_{0}\right) \text {. }
$$

Since

$$
\lim _{\lambda \rightarrow 0^{ \pm}} \frac{2}{\sqrt{\pi}} \int_{\varepsilon}^{\infty} \lambda e^{-t^{2} \lambda^{2}} d t= \pm 1,
$$

it follows that

$$
\lim _{u \rightarrow u_{0}^{-}} \eta_{\varepsilon}\left(D_{u}\right)=\eta_{\varepsilon}\left(D_{u_{0}}\right)+Q^{+}-Q^{-} .
$$

Recall that, by definition, $\operatorname{sf}\left(D_{a}, D_{u_{0}}\right)=Q^{-}$and

$$
\xi_{\varepsilon}\left(D_{u_{0}}\right)=\frac{\eta_{\varepsilon}\left(D_{u_{0}}\right)+\operatorname{dim} \operatorname{ker}\left(D_{u_{0}}\right)}{2}
$$

Therefore, the difference of equation (4.3) and equation (4.4) gives

$$
\operatorname{sf}\left(D_{a}, D_{u_{0}}\right)=\xi_{\varepsilon}\left(D_{u_{0}}\right)-\lim _{u \rightarrow u_{0}^{-}} \xi_{\varepsilon}\left(D_{u}\right)
$$

Similarly, $\operatorname{sf}\left(D_{u_{0}}, D_{b}\right)=\lim _{u \rightarrow u_{0}^{+}} \xi_{\varepsilon}\left(D_{u}\right)-\xi_{\varepsilon}\left(D_{u_{0}}\right)$. Thus we have

$$
\operatorname{sf}\left(D_{a}, D_{b}\right)=\lim _{u \rightarrow u_{0}^{+}} \xi_{\varepsilon}\left(D_{u}\right)-\lim _{u \rightarrow u_{0}^{-}} \xi_{\varepsilon}\left(D_{u}\right)
$$

With the above results combined, we have the following proposition. 


\section{Proposition 4.6.}

$$
\operatorname{sf}\left(D, g^{-1} D g\right)=\lim _{\varepsilon \rightarrow \infty} \frac{\varepsilon}{\sqrt{\pi}} \int_{0}^{1}{ }^{\mathrm{b}} \operatorname{Tr}\left(\dot{D}_{u} e^{-\varepsilon^{2} D_{u}^{2}}\right) d u .
$$

Proof. Let us cover $[0,1]$ by finitely many pointed gap intervals $\left[a_{i}, b_{i}\right], 0 \leq i \leq n$, with $u_{i} \in\left[a_{i}, b_{i}\right]$ such that $b_{i}=a_{i+1}$ with $u_{0}=a_{0}=0, u_{n}=b_{n}=1$ and $u_{j} \in\left(a_{j}, b_{j}\right)$ for $1 \leq j \leq n-1$. Then

$$
\begin{aligned}
\operatorname{sf}\left(D_{0}, D_{1}\right) & =\xi_{\varepsilon}\left(D_{1}\right)-\xi_{\varepsilon}\left(D_{0}\right)+\sum_{i} \lim _{u \rightarrow u_{i}^{+}} \xi_{\varepsilon}\left(D_{u}\right)-\lim _{u \rightarrow u_{i}^{-}} \xi_{\varepsilon}\left(D_{u}\right) \\
& =\xi_{\varepsilon}\left(D_{1}\right)-\xi_{\varepsilon}\left(D_{0}\right)-\frac{1}{2} \int_{0}^{1} \frac{d}{d u} \eta_{\varepsilon}\left(D_{u}\right) d u \\
& =\xi_{\varepsilon}\left(D_{1}\right)-\xi_{\varepsilon}\left(D_{0}\right)+\frac{\varepsilon}{\sqrt{\pi}} \int_{0}^{1}{ }^{\mathrm{b}} \operatorname{Tr}\left(\dot{D}_{u} e^{-\varepsilon^{2} D_{u}^{2}}\right) d u-\frac{1}{2} \int_{0}^{1} E_{\varepsilon}(u) d u .
\end{aligned}
$$

Notice that $\xi_{\varepsilon}\left(g^{-1} D g\right)=\xi_{\varepsilon}(D)$ and $\int_{0}^{1} E_{\varepsilon}(u) d u$ vanishes when $\varepsilon \rightarrow \infty$, hence the proposition follows.

\section{Large time limit}

In this section, we prove the equality

$$
\operatorname{sf}\left(D, g^{-1} D g\right)=\lim _{t \rightarrow \infty}\left\langle{ }^{\mathrm{b}} \mathrm{Ch}^{\bullet}(t \mathfrak{D}), \mathrm{Ch} \cdot(g)\right\rangle .
$$

This is the last step remaining to prove Theorem 3.1. We follow rather closely Getzler's proof for closed manifolds [Get93b].

Recall that we have

$$
\mathfrak{D}=\left(\begin{array}{cc}
0 & D \\
D & 0
\end{array}\right) \in{ }^{\mathrm{b}} \Psi^{1}\left(\widehat{M} ; \delta_{1}\right), \quad{ }^{\partial} \mathfrak{D}=\left(\begin{array}{cc}
0 & { }^{\partial} D \\
{ }^{\partial} D & 0
\end{array}\right) \in{ }^{\mathrm{b}} \Psi^{1}\left(\partial M ; S_{1}\right)
$$

and

$$
p=\left(\begin{array}{cc}
0 & g^{-1} \\
g & 0
\end{array}\right) \in C_{\exp }^{\infty}(\widehat{M}) \otimes \operatorname{End}\left(\mathbb{C}^{r \mid r}\right),
$$

with $\mathbb{C}^{r \mid r}=\left(\mathbb{C}^{r}\right)^{+} \oplus\left(\mathbb{C}^{r}\right)^{-}$being $\mathbb{Z}_{2}$-graded. Let us put

$$
\mathfrak{D}_{u}=(1-u) \mathfrak{D}-u p \mathfrak{D} p \in{ }^{\mathrm{b}} \Psi^{1}\left(\widehat{M} ; \oint_{1} \otimes_{s} \mathbb{C}^{r \mid r}\right)
$$

for $u \in[0,1]$, where $S_{1} \otimes_{s} \mathbb{C}^{r \mid r}$ is the super-tensor product of $S_{1}$ and $\mathbb{C}^{r \mid r}$. We see immediately that

$$
\begin{aligned}
\mathfrak{D}_{u} & =\left(\begin{array}{cc}
(1-u) \mathfrak{D}+u g^{-1} \mathfrak{D} g & 0 \\
0 & (1-u) \mathfrak{D}+u g \mathfrak{D} g^{-1}
\end{array}\right) \\
& =\left(\begin{array}{cc}
\mathfrak{D}+u g^{-1}[\mathfrak{D}, g] & 0 \\
0 & \mathfrak{D}+u g\left[\mathfrak{D}, g^{-1}\right]
\end{array}\right) .
\end{aligned}
$$


We write $\mathfrak{D}_{u, s}=\mathfrak{D}_{u}+s p\left(\operatorname{resp} .{ }^{\partial} \mathfrak{D}_{u, s}={ }^{\partial} \mathfrak{D}_{u}+s p\right)$, where $(u, s) \in[0,1] \times(-\infty, 0]$. Consider the superconnections $\mathbb{A}=d+\mathfrak{D}_{u, s}$ and ${ }^{\partial} \mathbb{A}=d+{ }^{\partial} \mathfrak{D}_{u, s}$, where $d$ is the standard de Rham differential on the parameter space $[0,1] \times(-\infty, 0]$. We have

$$
\begin{aligned}
\mathbb{A}^{2} & =\mathfrak{D}_{u}^{2}+s\left[\mathfrak{D}_{u}, p\right]+s^{2}+d u \dot{\mathfrak{D}}_{u}+d s p, \\
{ }^{\partial} \mathbb{A}^{2} & ={ }^{\partial} \mathfrak{D}_{u}^{2}+s\left[{ }^{2} \mathfrak{D}_{u},{ }^{\partial} p\right]+s^{2}+d u^{\partial} \dot{\mathfrak{D}}_{u}+d s^{\partial} p .
\end{aligned}
$$

Recall that the indicial family $I(D, \lambda)$ of $D$ is

$$
I(D, \lambda)={ }^{\partial} D+i \lambda c(v),
$$

where $v=d x$ is the normal cotangent vector and $c(v)$ is the Clifford multiplication of $v$, cf. [Get93b], Proposition 5.4. Therefore, we have

$$
\begin{aligned}
\mathrm{I}\left(\mathfrak{D}_{u}, \lambda\right) & ={ }^{\partial} \mathfrak{D}_{u}+i \lambda c(v), \\
\mathrm{I}\left(\mathfrak{D}_{u, s}, \lambda\right) & ={ }^{\partial} \mathfrak{D}_{u}+i \lambda c(v)+s^{\partial} p, \\
\mathrm{I}\left(d \mathfrak{D}_{u, s}, \lambda\right) & =d u^{\partial} \dot{\mathfrak{D}}_{u}+d s^{\partial} p, \\
\mathrm{I}\left(\mathfrak{D}_{u, s}^{2}, \lambda\right) & ={ }^{\partial} \mathfrak{D}_{u}^{2}+\lambda^{2}+s\left[{ }^{\partial} \mathfrak{D}_{u},{ }^{\partial} p\right]+s^{2} .
\end{aligned}
$$

Consider the Chern character of $\mathbb{A}$ defined by

$$
\operatorname{Ch}(\mathbb{A}):={ }^{\mathrm{b}} \operatorname{Str}_{(1)}\left(e^{-\mathbb{A}^{2}}\right) .
$$

Denote $\Gamma_{u}$ the contour $\{u\} \times[0, \infty)$ and $\gamma_{s}$ the contour $[0,1] \times\{s\}$. By Stoke's theorem, we have

$$
\int_{\Gamma_{1}} \operatorname{Ch}(\mathbb{A})-\int_{\Gamma_{0}} \operatorname{Ch}(\mathbb{A})+\int_{\gamma_{0}} \operatorname{Ch}(\mathbb{A})-\lim _{s \rightarrow \infty} \int_{\gamma_{s}} \operatorname{Ch}(\mathbb{A})=\int_{[0,1] \times[0, \infty)} d \operatorname{Ch}(\mathbb{A}) .
$$

5.1. Technical lemmas. In this section let us prove several technical lemmas. Notice that by definition, we have

$$
\begin{aligned}
\operatorname{Ch}(\mathbb{A})= & -d u \int_{0}^{1}{ }^{\mathrm{b}} \operatorname{Str}_{(1)}\left(e^{-\sigma\left(\mathfrak{D}_{u}^{2}+s\left[\mathfrak{D}_{u}, p\right]+s^{2}\right)} \dot{\mathfrak{D}}_{u} e^{-(1-\sigma)\left(\mathfrak{D}_{u}^{2}+s\left[\mathfrak{D}_{u}, p\right]+s^{2}\right)}\right) d \sigma \\
& -d s \int_{0}^{1}{ }^{1} \operatorname{Str}_{(1)}\left(e^{-\sigma\left(\mathfrak{D}_{u}^{2}+s\left[\mathfrak{D}_{u}, p\right]+s^{2}\right)} p e^{-(1-\sigma)\left(\mathfrak{D}_{u}^{2}+s\left[\mathfrak{D}_{u}, p\right]+s^{2}\right)}\right) d \sigma
\end{aligned}
$$

Lemma 5.1. We have

$$
\int_{\gamma_{0}} \operatorname{Ch}(\mathbb{A})=-\int_{0}^{1}{ }^{\mathrm{b}} \operatorname{Str}_{(1)}\left(\dot{\mathfrak{D}}_{u} e^{-\mathfrak{D}_{u}^{2}}\right) d u .
$$


Proof. Since

$$
\begin{aligned}
{ }^{\mathrm{b}} \operatorname{Str}_{(1)} & {\left[e^{-\sigma \mathfrak{D}_{u}^{2}}, \dot{\mathfrak{D}}_{u} e^{\left.-(1-\sigma) \mathfrak{D}_{u}^{2}\right]}\right.} \\
= & \frac{-1}{2 \pi i} \int_{-\infty}^{\infty} \operatorname{Str}_{(1)}\left(\frac{d\left(e^{-\sigma\left({ }^{\partial} \mathfrak{D}^{2}+\lambda^{2}\right)}\right)}{d \lambda} \partial \dot{\mathfrak{D}}_{u} e^{-(1-\sigma)\left({ }^{\partial} \mathfrak{D}^{2}+\lambda^{2}\right)}\right) d \lambda=0,
\end{aligned}
$$

it follows that

$$
\begin{aligned}
\int_{\Gamma_{0}} \operatorname{Ch}(\mathbb{A}) & =-\int_{0}^{1} d u\left(\int_{0}^{1}{ }^{\mathrm{b}} \operatorname{Str}_{(1)}\left(e^{-\sigma \mathfrak{D}_{u}^{2}} \dot{\mathfrak{D}}_{u} e^{-(1-\sigma) \mathfrak{D}_{u}^{2}}\right) d \sigma\right) \\
& =-\int_{0}^{1}{ }^{\mathrm{b}} \operatorname{Str}_{(1)}\left(\dot{\mathfrak{D}}_{u} e^{-\mathfrak{D}_{u}^{2}}\right) d u
\end{aligned}
$$

Lemma 5.2. We have

$$
\lim _{s \rightarrow \infty} \int_{\gamma_{s}} \operatorname{Ch}(\mathbb{A})=0 .
$$

Proof. First notice that a similar argument as that in Lemma 5.1 shows that

$$
\begin{gathered}
\int_{0}^{1}{ }^{\mathrm{b}} \operatorname{Str}_{(1)}\left(e^{-\sigma\left(\mathfrak{D}_{u}^{2}+s\left[\mathfrak{D}_{u}, p\right]+s^{2}\right)} p e^{-(1-\sigma)\left(\mathfrak{D}_{u}^{2}+s\left[\mathfrak{D}_{u}, p\right]+s^{2}\right)}\right) d \sigma \\
={ }^{\mathrm{b}} \operatorname{Str}_{(1)}\left(p e^{-\mathfrak{D}_{u}^{2}-s\left[\mathfrak{D}_{u}, p\right]-s^{2}}\right) .
\end{gathered}
$$

Using Duhamel's principle, we have

$$
\begin{aligned}
& { }^{\mathrm{b}} \operatorname{Str}_{(1)}\left(p e^{-\mathfrak{D}_{u}^{2}-s\left[\mathfrak{D}_{u}, p\right]-s^{2}}\right) \\
& \quad=\sum_{n=0}^{\infty} e^{-s^{2}}(-s)^{n} \int_{\Delta^{n}}{ }^{\mathrm{b}} \operatorname{Str}_{(1)}\left(p e^{-\sigma_{0} \mathfrak{D}_{u}^{2}}\left[\mathfrak{D}_{u}, p\right] e^{-\sigma_{1} \mathfrak{D}_{u}^{2}} \ldots\left[\mathfrak{D}_{u}, p\right] e^{-\sigma_{n} \mathfrak{D}_{u}^{2}}\right) d \sigma .
\end{aligned}
$$

The estimates in Section 2.2 show that

$$
\begin{gathered}
\left|\int_{\Delta^{n}}{ }^{\mathrm{b}} \operatorname{Str}_{(1)}\left(p e^{-\sigma_{0} \mathfrak{D}_{u}^{2}}\left[\mathfrak{D}_{u}, p\right] e^{-\sigma_{1} \mathfrak{D}_{u}^{2}} \ldots\left[\mathfrak{D}_{u}, p\right] e^{-\sigma_{n} \mathfrak{D}_{u}^{2}}\right) d \sigma\right| \\
\leq 2^{n}(n+1) \frac{\mathcal{K}_{1}^{n}+2 \mathcal{K}_{0}}{n !} \mathrm{b}\|p\|^{n+2}
\end{gathered}
$$

for some constants $\mathcal{K}_{0}$ and $\mathcal{K}_{1}$. In fact $\mathcal{K}_{0}$ and $\mathcal{K}_{1}$ can be chosen independent of $u$ since there is constant $\mathcal{C}$ such that

$$
\left|\operatorname{Tr}\left(e^{-\sigma \mathfrak{D}_{\mathbb{R}}^{2}}-e^{-\sigma \mathfrak{D}_{u}^{2}}\right)\right|_{(-\infty, 0] \times \partial M} \mid \leq \ell
$$

for all $\sigma, u \in[0,1]$ (cf. [LMP09], Proposition 3.1). Hence

$$
\left|{ }^{\mathrm{b}} \operatorname{Str}_{(1)}\left(p e^{-\mathfrak{D}_{u}^{2}-s\left[\mathfrak{D}_{u}, p\right]-s^{2}}\right)\right| \leq \mathcal{K}^{\prime} e^{-s^{2}+2 \mathcal{K}^{\mathrm{b}}\|p\| s}
$$

for some constants $\mathcal{K}$ and $\mathcal{K}^{\prime}$. Therefore $\int_{\gamma_{s}} \operatorname{Ch}(\mathbb{A})=O\left(e^{-s^{2} / 2}\right)$ as $s \rightarrow \infty$, hence the lemma follows. 
Lemma 5.3. We have

$$
\int_{\Gamma_{0}} \operatorname{Ch}(\mathbb{A})=-\int_{\Gamma_{1}} \operatorname{Ch}(\mathbb{A})=\frac{1}{2}\left\langle{ }^{b} \operatorname{Ch}^{\bullet}(\mathfrak{D}), \sum_{k=0}^{\infty} k ! \operatorname{Str}(p, \ldots, p)_{2 k+1}\right\rangle .
$$

Proof. When $u=0$, we have $\mathbb{A}^{2}=\mathfrak{D}^{2}+s[\mathfrak{D}, p]+s^{2}+d s p$. Using Duhamel's principle, we see that

$$
\begin{aligned}
& \left.\operatorname{Ch}(\mathbb{A})\right|_{\Gamma_{0}} \\
& \quad=\sum_{n=0}^{\infty}(-s)^{2 k+1} e^{-s^{2}} \int_{\Delta^{n}}{ }^{\mathrm{b}} \operatorname{Str}_{(1)}\left(e^{-\sigma_{0} \mathfrak{D}^{2}}[\mathfrak{D}, p] e^{-\sigma_{1} \mathfrak{D}^{2}} \ldots\right. \\
& \left.\quad e^{-\sigma_{i} \mathfrak{D}^{2}}(-d s p) e^{-\sigma_{i+1} \mathfrak{D}^{2}} \ldots[\mathfrak{D}, p] e^{-\sigma_{n} \mathfrak{D}^{2}}\right) d \sigma \\
& \quad \sum_{k=0}^{\infty} s^{2 k+1} e^{-s^{2}} d s \sum_{i=0}^{2 k+1} \mathrm{~b}\langle 1,[\mathfrak{D}, p], \ldots,[\mathfrak{D}, p], p,[\mathfrak{D}, p], \ldots,[\mathfrak{D}, p]\rangle \\
& =\sum_{k=0}^{\infty} s^{2 k+1} e^{-s^{2}} d s^{\mathrm{b}}\langle p,[\mathfrak{D}, p], \ldots,[\mathfrak{D}, p]\rangle_{2 k+1},
\end{aligned}
$$

where we have used the fact (cf. [Get93a], Lemma 6.3 (2))

$$
\sum_{i=0}^{2 k+1} \mathrm{~b}\langle 1,[\mathfrak{D}, p], \ldots,[\mathfrak{D}, p], p,[\mathfrak{D}, p], \ldots,[\mathfrak{D}, p]\rangle={ }^{\mathrm{b}}\langle p,[\mathfrak{D}, p], \ldots,[\mathfrak{D}, p]\rangle_{2 k+1} \text {. }
$$

It follows that

$$
\int_{\Gamma_{0}} \operatorname{Ch}(\mathbb{A})=\frac{1}{2}\left\langle{ }^{b} \mathrm{Ch}^{\bullet}(\mathfrak{D}), \sum_{k=0}^{\infty} k ! \operatorname{Str}(p, \ldots, p)_{2 k+1}\right\rangle .
$$

When $u=1$, we have $\mathbb{A}^{2}=\mathfrak{D}_{1}^{2}+s\left[\mathfrak{D}_{1}, p\right]+s^{2}+d s p$. Since $\mathfrak{D}_{1}=-p \mathfrak{D} p$, we see that

$$
\left[\mathfrak{D}_{1}, p\right]=-[\mathfrak{D}, p] \quad \text { and } \quad e^{-\mathfrak{D}_{1}^{2}}=p e^{-\mathfrak{D}^{2}} p .
$$

Furthermore, we notice that $p[\mathfrak{D}, p] p=[\mathfrak{D}, p]$. Combining these with a calculation similar to that in equation (5.2) with $\mathfrak{D}$ replaced by $\mathfrak{D}_{1}$, we see that

$$
\int_{\Gamma_{1}} \operatorname{Ch}(\mathbb{A})=-\frac{1}{2}\left\langle{ }^{b} \mathrm{Ch}^{\bullet}\left(\mathfrak{D}_{1}\right), \sum_{k=0}^{\infty} k ! \operatorname{Str}(p, \ldots, p)_{2 k+1}\right\rangle
$$

Lemma 5.4. We have

$$
d \operatorname{Ch}(\mathbb{A})=-{ }^{\mathrm{b}} \operatorname{Str}\left[\mathfrak{D}_{u, s}, e^{-\mathbb{A}^{2}}\right]=\operatorname{Str}_{(2)}\left(e^{-{ }^{\partial} \mathbb{A}^{2}}\right) .
$$

Proof. Since $\left[\mathbb{A}, e^{-\mathbb{A}^{2}}\right]=0$, we have

$$
d{ }^{\mathrm{b}} \operatorname{Str}_{(1)}\left(e^{-\mathbb{A}^{2}}\right)={ }^{\mathrm{b}} \operatorname{Str}_{(1)}\left[d, e^{-\mathbb{A}^{2}}\right]=-{ }^{\mathrm{b}} \operatorname{Str}_{(1)}\left[\mathfrak{D}_{u, s}, e^{-\mathbb{A}^{2}}\right] .
$$


It follows that

$$
\begin{aligned}
d \operatorname{Ch}(\mathbb{A}) & =\frac{1}{2 \pi i} \int_{-\infty}^{\infty} \operatorname{Str}_{(1)}\left(\frac{d \mathrm{I}\left(\mathfrak{D}_{u, s}, \lambda\right)}{d \lambda} \mathrm{I}\left(e^{-\mathbb{A}^{2}}, \lambda\right)\right) d \lambda \\
& =\frac{1}{2 \pi i} \int_{-\infty}^{\infty} \operatorname{Str}_{(1)}\left(i c(v) \mathrm{I}\left(e^{-\mathbb{A}^{2}}, \lambda\right)\right) d \lambda \\
& =\frac{1}{\sqrt{\pi}} \int_{-\infty}^{\infty} \operatorname{Str}_{(2)}\left(\mathrm{I}\left(e^{-\mathbb{A}^{2}}, \lambda\right)\right) d \lambda \\
& =\frac{1}{\sqrt{\pi}} \int_{-\infty}^{\infty} e^{-\lambda^{2}} d \lambda \operatorname{Str}_{(2)}\left(e^{-\partial^{\mathbb{A}^{2}}}\right) \\
& =\operatorname{Str}_{(2)}\left(e^{-{ }^{\partial} \mathbb{A}^{2}}\right) .
\end{aligned}
$$

By Duhamel's principle, the 2-form components in $\operatorname{Str}_{(2)}\left(e^{-{ }^{\partial} \mathbb{A}^{2}}\right)$ can be expanded as

$$
\begin{gathered}
\sum_{k=2}^{\infty} \sum_{1 \leq i<j \leq k}(-s)^{k-2} e^{-s^{2}}\langle 1,\left[{ }^{\partial} \mathfrak{D}_{u}, p\right], \ldots,\left[{ }^{\partial} \mathfrak{D}_{u}, p\right], \underbrace{p}_{i-\text { th }},\left[{ }^{\partial} \mathfrak{D}_{u}, p\right], \ldots \\
\ldots,\left[{ }^{\partial} \mathfrak{D}_{u}, p\right], \underbrace{\underbrace{\partial \mathfrak{D}_{u}}}_{j \text {-th }},\left[{ }^{\partial} \mathfrak{D}_{u}, p\right], \ldots,\left[{ }^{\partial} \mathfrak{D}_{u}, p\right]\rangle_{u} \mathrm{~d} u \mathrm{~d} s \\
-\sum_{k=2}^{\infty} \sum_{1 \leq i<j \leq k}(-s)^{k-2} e^{-s^{2}}\langle 1,\left[{ }^{\partial} \mathfrak{D}_{u}, p\right], \ldots,\left[{ }^{\partial} \mathfrak{D}_{u}, p\right], \underbrace{\partial \mathfrak{D}_{u}}_{i-\text { th }},\left[{ }^{\partial} \mathfrak{D}_{u}, p\right], \ldots \\
\ldots,\left[{ }^{\partial} \mathfrak{D}_{u}, p\right], \underbrace{p}_{j \text {-th }},\left[{ }^{\partial} \mathfrak{D}_{u}, p\right], \ldots,\left[{ }^{\partial} \mathfrak{D}_{u}, p\right]\rangle_{u} \mathrm{~d} u \mathrm{~d} s,
\end{gathered}
$$

where

$$
\left\langle A_{0}, \ldots, A_{n}\right\rangle_{u}=\int_{\Delta^{n}}^{{ }^{\mathrm{b}}} \operatorname{Str}_{(2)}\left(A_{0} e^{-\sigma_{0}{ }^{\partial} \mathfrak{D}_{u}^{2}} A_{1} e^{-\sigma_{1}{ }^{\partial} \mathfrak{D}_{u}^{2}} \ldots A_{n} e^{-\sigma_{n}{ }^{\partial} \mathfrak{D}_{u}^{2}}\right) d \sigma
$$

Recall that (cf. [GS89], Lemma 2.2)

$$
\begin{aligned}
& \left\langle A_{0}, \ldots, A_{n}\right\rangle_{u} \\
& \quad=\sum_{i=0}^{n}(-1)^{\left(\left|A_{0}\right|+\cdots+\left|A_{i-1}\right|\right)\left(\left|A_{i}\right|+\cdots+\left|A_{n}\right|\right)}\left\langle 1, A_{i}, \ldots, A_{n}, A_{0}, \ldots, A_{i-1}\right\rangle_{u} .
\end{aligned}
$$

Since ${ }^{\partial} \mathfrak{D}_{u},{ }^{\partial} \dot{\mathfrak{D}}_{u}$ and $p$ are of odd degree and $\left[{ }^{\partial} \mathfrak{D}_{u}, p\right]$ is of even degree, one has

$$
\begin{aligned}
(5.3)= & \sum_{k=2}^{\infty} \sum_{i=1}^{k-1}(-s)^{k-2} e^{-s^{2}}\langle p,\left[{ }^{\partial} \mathfrak{D}_{u}, p\right], \ldots,\left[{ }^{\partial} \mathfrak{D}_{u}, p\right], \underbrace{\partial \dot{\mathfrak{D}}_{u}}_{i \text {-th }},\left[{ }^{\partial} \mathfrak{D}_{u}, p\right], \ldots \\
& \left.\ldots,\left[{ }^{\partial} \mathfrak{D}_{u}, p\right]\right\rangle_{u} \mathrm{~d} u \mathrm{~d} s .
\end{aligned}
$$


Let us define

$$
\widetilde{\mathrm{Ch}}^{n}\left({ }^{\partial} \mathfrak{D}_{u}, V\right)\left(a_{0}, \ldots, a_{n}\right)=\iota(V)\left\langle a_{0},\left[{ }^{\partial} \mathfrak{D}_{u}, a_{1}\right], \ldots,\left[{ }^{\partial} \mathfrak{D}_{u}, a_{n}\right]\right\rangle_{u},
$$

where

$$
\iota(V)\left\langle A_{0}, \ldots, A_{n}\right\rangle_{u}:=\sum_{0 \leq i \leq n}(-1)^{|V|\left(\left|A_{0}\right|+\cdots+\left|A_{i}\right|\right)}\left\langle A_{0}, \ldots, A_{i}, V, A_{i+1}, \ldots, A_{n}\right\rangle_{u} .
$$

Then the calculation above shows that

$$
\operatorname{Str}_{(2)}\left(e^{-{ }^{\partial} \mathbb{A}^{2}}\right)=-\sum_{k=0}^{\infty}(-s)^{k} e^{-s^{2}}\left\langle\widetilde{C h}^{k}\left({ }^{\partial} \mathfrak{D}_{u},{ }^{\partial \dot{D}_{u}}\right), \operatorname{Str}(p, \ldots, p)_{k}\right\rangle_{u} \mathrm{~d} u \mathrm{~d} s .
$$

We summarize this in the following lemma.

Lemma 5.5. We have

$$
\int_{[0,1] \times[0, \infty)} \operatorname{Str}_{(2)}\left(e^{-{ }^{\partial} \mathbb{A}^{2}}\right)=\frac{1}{2}\left\langle\int_{0}^{1} \widetilde{\operatorname{Ch}} \cdot\left({ }^{\partial} \mathfrak{D}_{u},{ }^{\partial} \dot{\mathfrak{D}}_{u}\right) \mathrm{d} u, \sum_{k=0}^{\infty} k ! \operatorname{Str}(p, \ldots, p)_{2 k+1}\right\rangle .
$$

Proof. Notice that $\operatorname{Str}(p, \ldots, p)_{k}=0$ for $k$ even, and

$$
\int_{0}^{\infty} s^{2 k+1} e^{-s^{2}} \mathrm{~d} s=\frac{k !}{2} .
$$

5.2. Large time limit. Recall that ${ }^{\partial} D$ is invertible and $g \in U_{k}\left(C^{\infty}(N)\right)$ is a unitary such that $\left\|\left[{ }^{\partial} D,{ }^{\partial} g\right]\right\|<\lambda$ with $\lambda$ the lowest nonzero eigenvalue of $\left|{ }^{\partial} D\right|$. In the following, we also write $g$ for ${ }^{g} g$ if there is no confusion. Notice that

$$
\left\|\left[{ }^{\partial} D, g^{-1}\right]\right\|=\left\|-g^{-1}\left[{ }^{\partial} D, g\right] g^{-1}\right\| \leq\left\|\left[{ }^{\partial} D, g\right]\right\|,
$$

and similarly $\left\|\left[{ }^{\partial} D, g\right]\right\| \leq\left\|\left[{ }^{\partial} D, g^{-1}\right]\right\|$. Hence $\left\|\left[{ }^{\partial} D, g^{-1}\right]\right\|=\left\|\left[{ }^{\partial} D, g\right]\right\|$.

Lemma 5.6. Let $\mathbb{A}_{t}=d+t \mathfrak{D}_{u, s}$. Then

$$
\lim _{t \rightarrow \infty} \int_{[0,1] \times[0, \infty)} d \operatorname{Ch}\left(\mathbb{A}_{t}\right)=0
$$

Proof. Notice that

$$
\begin{aligned}
& \left|{ }^{\partial} D+u g^{-1}\left[{ }^{\partial} D, g\right]\right| \geq\left|{ }^{\partial} D\right|-u\left\|g^{-1}\left[{ }^{\partial} D, g\right]\right\| \geq \lambda-u\left\|\left[{ }^{\partial} D, g\right]\right\|, \\
& \left|{ }^{\partial} D+u g\left[{ }^{\partial} D, g^{-1}\right]\right| \geq \lambda-u\left\|\left[{ }^{\partial} D, g\right]\right\| .
\end{aligned}
$$

When $u=1,{ }^{\partial} D+u g^{-1}\left[{ }^{\partial} D, g\right]=g^{-1} \partial D g$. The lowest eigenvalue of $\left|g^{-1}{ }^{\partial} D g\right|$ is also $\lambda$ since $g$ is a unitary. Therefore, a similar argument as above shows that

$$
\begin{aligned}
& \left|{ }^{\partial} D+u g^{-1}\left[{ }^{\partial} D, g\right]\right| \geq \lambda-(1-u)\left\|\left[{ }^{\partial} D, g\right]\right\|, \\
& \left|{ }^{\partial} D+u g\left[{ }^{\partial} D, g^{-1}\right]\right| \geq \lambda-(1-u)\left\|\left[{ }^{\partial} D, g\right]\right\| .
\end{aligned}
$$


Thus $\left|{ }^{\partial} \mathfrak{D}_{u}\right|$ is bounded below by

$$
\lambda_{u}:=\max \left\{\lambda-u\left\|\left[{ }^{\partial} D, g\right]\right\|, \lambda-(1-u)\left\|\left[{ }^{\partial} D, g\right]\right\|\right\} .
$$

Then there exists a constant $C$ such that

$$
\operatorname{Tr}\left(e^{-t^{2 \partial} \mathfrak{D}_{u}^{2}}\right) \leq C e^{-t^{2} \lambda_{u}^{2}}
$$

for all $t \geq 1$. where we may take $C=\sup _{u \in[0,1]} e^{\lambda_{u}^{2}} \operatorname{Tr}\left(e^{-\partial \mathfrak{D}_{u}^{2}}\right)$, cf. [GS89], Theorem C. One also notices that $\left\|\left[{ }^{\partial} \mathfrak{D}_{u}, p\right]\right\|=(1-2 u)\left\|\left[{ }^{\partial} \mathfrak{D}, p\right]\right\|<\lambda_{u}$. Therefore we have

$$
\begin{aligned}
\mid t^{2 k+2} & \left\langle p,\left[{ }^{\partial} \mathfrak{D}_{u}, p\right], \ldots,\left[{ }^{\partial} \mathfrak{D}_{u}, p\right],{ }^{\partial} \dot{\mathfrak{D}}_{u},\left[{ }^{\partial} \mathfrak{D}_{u}, p\right], \ldots,\left[{ }^{\partial} \mathfrak{D}_{u}, p\right]\right\rangle_{u, 2 k+1} \mid \\
& \leq \frac{1}{(2 k) !} t^{2 k+2} \operatorname{Tr}\left(e^{-t^{2 \partial} \mathfrak{D}_{u}^{2}}\right)\|p\| \cdot\left\|\left[{ }^{\partial} \mathfrak{D}_{u}, p\right]\right\|^{2 k+1}\left\|\dot{\mathfrak{D}}_{u}\right\| \\
& \leq \frac{C}{(2 k) !} t^{2 k+2} e^{-t^{2} \lambda_{u}^{2}}\left\|\left[{ }^{\partial} \mathfrak{D}_{u}, p\right]\right\|^{2 k+1}\left\|p\left[{ }^{\partial} \mathfrak{D}, p\right]\right\| .
\end{aligned}
$$

Hence

$$
\begin{aligned}
\left|\int_{[0,1] \times[0, \infty)} d \operatorname{Ch}\left(\mathbb{A}_{t}\right)\right| & =\left|\int_{[0,1] \times[0, \infty)} \operatorname{Str}_{(2)}\left(e^{-{ }^{\partial} \mathbb{A}_{t}^{2}}\right)\right| \\
& =\left|\left\langle\int_{0}^{1} \widetilde{\operatorname{Ch}}^{\cdot}\left(t^{\partial} \mathfrak{D}_{u}, t^{\partial} \dot{\mathfrak{D}}_{u}\right) \mathrm{d} u, \sum_{k=0}^{\infty} k ! \operatorname{Str}(p, \ldots, p)_{2 k+1}\right\rangle\right| \\
& \leq t \cdot\left\|p\left[{ }^{\partial} \mathfrak{D}, p\right]\right\| \int_{0}^{1} \sum_{k=1}^{\infty} \frac{C}{k !} e^{-t^{2} \lambda_{u}^{2}}\left(\left\|\left[{ }^{\partial} \mathfrak{D}_{u}, p\right]\right\| \cdot t\right)^{2 k+1} d u \\
& \leq t^{2} C^{\prime} \int_{0}^{1} e^{\left(\left\|\left[^{\partial} \mathfrak{D}_{u}, p\right]\right\|^{2}-\lambda_{u}^{2}\right) t^{2}} d u,
\end{aligned}
$$

where the last term goes to 0 when $t \rightarrow \infty$. This finishes the proof.

Lemma 5.7. If $g \in U_{k}\left(C^{\infty}(N)\right)$ is a unitary such that $\left\|\left[{ }^{\partial} D,{ }^{\partial} g\right]\right\|<\lambda$ with $\lambda$ the lowest nonzero eigenvalue of $\left|{ }^{\partial} D\right|$, then

$$
\lim _{t \rightarrow \infty}\left\langle{ }^{\mathrm{b}} \mathrm{Ch}^{\bullet}(t \mathfrak{D}), \mathrm{Ch} \cdot(g)\right\rangle=-\lim _{t \rightarrow \infty}\left\langle{ }^{\mathrm{b}} \mathrm{Ch}^{\bullet}(t \mathfrak{D}), \mathrm{Ch} \cdot\left(g^{-1}\right)\right\rangle .
$$

Proof. Recall equation (3.1):

$$
(d-b-B)^{\mathrm{b}} \mathrm{Ch}^{\bullet}(\mathfrak{D}, t)=\mathrm{Ch}^{\bullet}\left({ }^{\partial} \mathfrak{D}, t\right) .
$$

Since Ch. $(g)+$ Ch. $_{\bullet}\left(g^{-1}\right)-2 C_{\bullet}(1)=(b+B)$ Tch. $(h)$, where $h$ is a smooth path 
of unitaries connecting $\left(\begin{array}{ll}1 & 0 \\ 0 & 1\end{array}\right)$ and $\left(\begin{array}{cc}g & 0 \\ 0 & g^{-1}\end{array}\right)$ (cf. Section 1.6), we have

$$
\begin{aligned}
\lim _{t \rightarrow \infty}\left\langle{ }^{\mathrm{b}} \mathrm{Ch}^{\bullet}(t \mathfrak{D}), \mathrm{Ch}_{\bullet}(g)+\mathrm{Ch}_{\bullet}\left(g^{-1}\right)\right\rangle & =\lim _{t \rightarrow \infty}\left\langle{ }^{\mathrm{b}} \mathrm{Ch}^{\bullet}(t \mathfrak{D}), \mathrm{Ch}_{\bullet}(g)+\mathrm{Ch}_{\bullet}\left(g^{-1}\right)-2 \mathrm{Ch} \cdot(1)\right\rangle \\
= & \lim _{t \rightarrow \infty}\left\langle(b+B)^{\mathrm{b}} \mathrm{Ch}^{\bullet}(t \mathfrak{D}), \mathrm{Tch}_{\bullet}(h)\right\rangle \\
& =-\lim _{t \rightarrow \infty}\left\langle\mathrm{Ch}^{\bullet}\left(t^{\mathrm{d}} \mathfrak{D}\right), \mathrm{Tch}_{\bullet}(h)\right\rangle .
\end{aligned}
$$

Now a similar argument (without the presence of the parameter $u$ ) as that in Lemma 5.6 above shows that

$$
\lim _{t \rightarrow \infty}\left\langle\mathrm{Ch}^{\bullet}\left(t^{\partial} \mathfrak{D}\right), \operatorname{Tch}_{\bullet}(h)\right\rangle=0 .
$$

Hence the lemma follows.

Proposition 5.8. We have

$$
\operatorname{sf}\left(D, g^{-1} D g\right)=\lim _{t \rightarrow \infty}\left\langle{ }^{\mathrm{b}} \mathrm{Ch}^{\bullet}(t \mathfrak{D}), \mathrm{Ch} \cdot(g)\right\rangle
$$

Proof. Notice that

$$
\begin{aligned}
& \mathfrak{D}_{u}=\left(\begin{array}{cc}
\mathfrak{D}+u g^{-1}[\mathfrak{D}, g] & 0 \\
0 & \mathfrak{D}+u g\left[\mathfrak{D}, g^{-1}\right]
\end{array}\right) \\
& =\left(\begin{array}{cccc}
0 & D+u g^{-1}[D, g] & & \\
D+u g^{-1}[D, g] & 0 & 0 & D+u g\left[D, g^{-1}\right]
\end{array}\right) \text {. }
\end{aligned}
$$

Hence

$$
\begin{aligned}
\int_{0}^{1}{ }^{\mathrm{b}} \operatorname{Str}_{(1)}\left(\dot{\mathfrak{D}}_{u} e^{-\mathfrak{D}_{u}^{2}}\right) d u= & \frac{1}{\sqrt{\pi}} \int_{0}^{1}{ }^{\mathrm{b}} \operatorname{Tr}\left(g^{-1}[D, g] e^{-\left(D+u g^{-1}[D, g]\right)^{2}}\right) d u \\
& -\frac{1}{\sqrt{\pi}} \int_{0}^{1}{ }^{\mathrm{b}} \operatorname{Tr}\left(g\left[D, g^{-1}\right] e^{-\left(D+u g\left[D, g^{-1}\right]\right)^{2}}\right) d u .
\end{aligned}
$$

It follows from Theorem 4.6 that

$$
\begin{aligned}
\lim _{t \rightarrow \infty} \int_{0}^{1}{ }^{\mathrm{b}} \operatorname{Str}_{(1)}\left(t \dot{\mathfrak{D}}_{u} e^{-t^{2} \mathfrak{D}_{u}^{2}}\right) d u & =\operatorname{sf}\left(D, g^{-1} D g\right)-\operatorname{sf}\left(D, g D g^{-1}\right) \\
& =2 \operatorname{sf}\left(D, g^{-1} g D\right)
\end{aligned}
$$

since $\operatorname{sf}\left(D, g D g^{-1}\right)=-\operatorname{sf}\left(D, g^{-1} D g\right)$. 
Now by applying Lemma 5.2, 5.3 and 5.1 to equation (5.1), we have

$$
\begin{gathered}
-\left\langle{ }^{\mathrm{b}} \mathrm{Ch}^{\bullet}(t \mathfrak{D}), \sum_{k=0}^{\infty} k ! \operatorname{Str}(p, \ldots, p)_{2 k+1}\right\rangle-\int_{0}^{1}{ }^{\mathrm{b}} \operatorname{Str}_{(1)}\left(t \dot{\mathfrak{D}}_{u} e^{-t^{2} \mathfrak{D}_{u}^{2}}\right) d u \\
=\int_{[0,1] \times[0, \infty)} d \operatorname{Ch}\left(\mathbb{A}_{t}\right) .
\end{gathered}
$$

Therefore,

$$
\begin{aligned}
\lim _{t \rightarrow \infty} & \int_{0}^{1}{ }^{\mathrm{b}} \operatorname{Str}_{(1)}\left(t \dot{\mathfrak{D}}_{u} e^{-t^{2} \mathfrak{D}_{u}^{2}}\right) d u \\
& =\lim _{t \rightarrow \infty}\left\langle{ }^{\mathrm{b}} \mathrm{Ch}^{\bullet}(t \mathfrak{D}), \mathrm{Ch}_{\bullet}(g)-\mathrm{Ch}_{\bullet}\left(g^{-1}\right)\right\rangle-\lim _{t \rightarrow \infty} \int_{[0,1] \times[0, \infty)} d \operatorname{Ch}\left(\mathbb{A}_{t}\right) .
\end{aligned}
$$

It follows from Lemma 5.6 that

$$
2 \operatorname{sf}\left(D, g^{-1} D g\right)=\lim _{t \rightarrow \infty}\left\langle{ }^{\mathrm{b}} \mathrm{Ch}^{\bullet}(t \mathfrak{D}), \mathrm{Ch}_{\bullet}(g)-\mathrm{Ch}_{\bullet}\left(g^{-1}\right)\right\rangle .
$$

Now applying Lemma 5.7, we have

$$
\operatorname{sf}\left(D, g^{-1} D g\right)=\lim _{t \rightarrow \infty}\left\langle{ }^{\mathrm{b}} \mathrm{Ch}^{\bullet}(t \mathfrak{D}), \mathrm{Ch} \cdot(g)\right\rangle .
$$

\section{References}

[BF90] J. Block and J. Fox, Asymptotic pseudodifferential operators and index theory. In Geometric and topological invariants of elliptic operators (Brunswick, ME, 1988), Contemp. Math. 105, Amer. Math. Soc., Providence, RI, 1990, 1-32. Zbl 0704.58048 MR 1047274

[BBLP05] B. Booss-Bavnbek, M. Lesch, and J. Phillips, Unbounded Fredholm operators and spectral flow. Canad. J. Math. 57 (2005), 225-250. Zbl 1085.58018 MR 2124916

[CM90] A. Connes and H. Moscovici, Cyclic cohomology, the Novikov conjecture and hyperbolic groups. Topology 29 (1990), 345-388. Zbl 0759.58047 MR 1066176

[DZ06] X. Dai and W. Zhang, An index theorem for Toeplitz operators on odd-dimensional manifolds with boundary. J. Funct. Anal. 238 (2006), 1-26. Zbl 1114.58011 MR 2234121

[Get83] E. Getzler, Pseudodifferential operators on supermanifolds and the AtiyahSinger index theorem. Comm. Math. Phys. 92 (1983), 163-178. Zbl 0543.58026 MR 728863

[Get93a] E. Getzler, Cyclic homology and the Atiyah-Patodi-Singer index theorem. In Index theory and operator algebras (Boulder, CO, 1991), Contemp. Math. 148, Amer. Math. Soc., Providence, RI 1993, 19-45. Zbl 0798.46049 MR 1228498

[Get93b] E. Getzler, The odd Chern character in cyclic homology and spectral flow. Topology 32 (1993), 489-507. Zbl 0801.46088 MR 1231957 
[GS89] E. Getzler and A. Szenes, On the Chern character of a theta-summable Fredholm module. J. Funct. Anal. 84 (1989), 343-357. Zbl 0686.46044 MR 1001465

[JLO88] A. Jaffe, A. Lesniewski, and K. Osterwalder, Quantum $K$-theory. I. The Chern character. Comm. Math. Phys. 118 (1988), 1-14. Zbl 0656.58048 MR 954672

[Kat95] T. Kato, Perturbation theory for linear operators. Classics Math., Springer-Verlag, Berlin 1995. Zbl 0836.47009 MR 1335452

[KL04] P. Kirk and M. Lesch, The $\eta$-invariant, Maslov index, and spectral flow for Diractype operators on manifolds with boundary. Forum Math. 16 (2004), 553-629. Zbl 1082.58021 MR 2044028

[LMP09] M. Lesch, H. Moscovici, and M. J. Pflaum, Relative pairing in cyclic cohomology and divisor flows. J. K-Theory 3 (2009), 359-407. Zbl 1175.46066 MR 2496452

[Loy05] P. Loya, Dirac operators, boundary value problems, and the $b$-calculus. In Spectral geometry of manifolds with boundary and decomposition of manifolds, Contemp. Math. 366, Amer. Math. Soc., Providence, RI, 2005, 241-280. Zbl 1065.58017 MR 2114491

[Me193] R. B. Melrose, The Atiyah-Patodi-Singer index theorem. Res. Notes Math. 4, A K Peters Ltd., Wellesley, MA, 1993. Zbl 0796.58050 MR 1348401

[Wu93] F. Wu, The Chern-Connes character for the Dirac operator on manifolds with boundary. K-Theory 7 (1993), 145-174. Zbl 0787.58041 MR 1235286

Received November 22, 2010; revised May 10, 2011

Z. Xie, Department of Mathematics, Vanderbilt University, 1326 Stevenson Center, Nashville, TN 37240, U.S.A.

E-mail: zhizhang.xie@vanderbilt.edu 